\title{
ON APPROXIMATE CONTROLLABILITY OF GENERALIZED KDV SOLITONS
}

\author{
CLAUDIO MUÑOZ*
}

\begin{abstract}
We consider the approximate control of solitons in generalized Korteweg-de Vries equations. By introducing a suitable internal bilinear control on the equation, we prove that any soliton is approximate null controllable, and moreover, any soliton can be accelerated to any particular positive velocity, after a suitable large amount of time. Precise estimates on the error terms and the rate of decay in the approximate null controllability result are also given. Our method introduces a new insight on the control of nonlinear objects, from the point of view of interaction and collision problems for nonlinear dispersive equations, recently developed by Y. Martel and F. Merle [23, 24]. It can be applied in principle, to several other models with soliton solutions.
\end{abstract}

AMS subject classifications. Primary 35Q51, 35Q53; Secondary 37K10, 37K40

Key words. gKdV equation, stabilization, approximate controllability, soliton

1. Introduction. In this paper we consider the problem of controlling solitons of subcritical, generalized Korteweg-de Vries equations (gKdV). More precisely, we look for an internal control $f=f(t, x)$ applied to modify the dynamics of generalized solitons of the equation

$$
u_{t}+\left(u_{x x}+u^{p}\right)_{x}=f, \quad p=2,3 \text { or } 4 .
$$

Here $u=u(t, x)$ is a real-valued function, and $(t, x) \in \mathbb{R}^{2}$. When $p=2$ and $f \equiv 0$ (1.1) is the well-known integrable Korteweg-de Vries equation (KdV).

Additionally, if $f \equiv 0$, equation (1.1) is a standard gKdV equation. It has special solitary wave solutions called solitons 1 of the form

$$
u(t, x)=Q_{c}(x-c t), \quad Q_{c}(s):=c^{\frac{1}{p-1}} Q(\sqrt{c} s), \quad c>0,
$$

with

$$
Q(s):=\left[\frac{p+1}{2 \cosh ^{2}\left(\frac{1}{2}(p-1) s\right)}\right]^{\frac{1}{p-1}} .
$$

The parameter $c>0$ is usually denoted as the scaling, or in a equivalent way, as the velocity of the soliton. Inserting the previous profile in (1.1) (recall that $f \equiv 0$ ), one has that $Q_{c}>0$ satisfies the nonlinear ODE

$$
Q_{c}^{\prime \prime}-c Q_{c}+Q_{c}^{p}=0, \quad Q_{c} \in H^{1}(\mathbb{R}) .
$$

Moreover, standard conservation laws for (1.1) at the $H^{1}$-level are the mass

$$
M[u](t):=\frac{1}{2} \int_{\mathbb{R}} u^{2}(t, x) d x=M[u](0),
$$

\footnotetext{
*Laboratoire de Mathématiques d'Orsay, Bât. 425, Université Paris-Sud 91405 Orsay Cedex France, email: claudio.munoz@math.u-psud.fr

${ }^{1}$ Strictly speaking, we should say solitary waves instead of solitons, but since we are not dealing with integrability issues, we will adopt the denomination soliton. This misunderstanding has spread out through the dispersive models community.
} 
and energy

$$
E[u](t):=\frac{1}{2} \int_{\mathbb{R}} u_{x}^{2}(t, x) d x-\frac{1}{p+1} \int_{\mathbb{R}} u^{p+1}(t, x) d x=E[u](0) .
$$

A satisfactory Cauchy theory is also present at the $H^{1}$ level of regularity, see e.g. Kenig-Ponce-Vega [14. The condition on $p$ is necessary to get global existence for general $H^{1}$-data, cf. the paper by Martel and Merle 21] for the critical case $p=5$. When $p>5$, solitons are unstable [4].

The control problem for the non inviscid KdV equation in a finite length interval has been extensively studied in the last twenty years, starting from the works of Zhang [1], Russell and Zhang in [37, 36, and [38, for a system with periodic boundary conditions and with an internal control. For the case of a boundary control, see [38 and 39. Concerning the non periodic framework, Rosier studied [33] the controllability of the KdV equation posed on a finite interval of $(0, L)$, under homogeneous Dirichlet boundary conditions and a control acting on the Neumann data at the right end-point of the interval. In particular, Rosier showed that if the length $L$ does not belong to a set of critical values, both the associated linear and the nonlinear systems are exactly controllable. When $L$ is critical, the linear system is not controllable because of the existence of a finite-dimensional subspace of unreachable states. In this case, the exact controllability of the $\mathrm{KdV}$ equation, in the case of critical domains, has been proven by Coron-Crépeau [6, Cerpa [7, and Cerpa-Crépeau [8]. Concerning the exact boundary control problem in the half-line, see e.g. the work of Rosier [34].

In this paper, unlike the previous results, we are interested in the study of a control problem associated to a given gKdV soliton posed on the real line. The main motivation of our problem will come from the fact that usual techniques from control theory cannot handle some controllability problems posed in unbounded domains, and even worse, the emergency of very particular nonlinear solutions cannot be treated using just linear techniques.

Let us explain in more detail the problem. Given an initial datum $u\left(t_{0}, x\right)=$ $u_{0}(x)=Q_{c}\left(x-c t_{0}-x_{0}\right)$ of soliton type, our objective is to introduce a control $f$ in the gKdV equation (1.1), during an interval of time $[0, T]$, with the purpose of accelerating the soliton to a new soliton state, with a different (positive) velocity. With no loss of generality, we can assume $t_{0}=x_{0}=0$ and that the initial velocity satisfies $c=1$. In other words, our goal is to determine sufficient conditions on $f$ to ensure that, given any final scaling $c_{f}>0$, the system (1.1) with initial datum $u_{0}$ evolves to a soliton of the form $Q_{c_{f}}$, up to some small error terms, in a suitable time of interaction $T>0$. Moreover, we also want to estimate the position of the soliton, compared with the theoretically expected position $\sim c_{f} T$.

We will assume that the interior control $f$ is given by the bilinear control (or feedback law)

$$
f(t, x)=a(t, x) u(t, x),
$$

with $a$ an internal potential satisfying the a priori assumptions

$$
a(t, \cdot) \in C^{3}(\mathbb{R}) \cap L^{2}(\mathbb{R}) \cap L^{\infty}(\mathbb{R}) .
$$

In other words, our control will use some explicit information of the soliton solution at each time, such as the scaling and position parameters. This problem has been also 
considered in a more physical context by Kaup-Newell [13], Grimshaw [10, Ko-Kuehl [15], and Lochak [18.

Therefore, in what follows, we consider the initial value problem

$$
\left\{\begin{array}{l}
u_{t}+\left(u_{x x}+u^{p}\right)_{x}=a(t, x) u \text { in } \mathbb{R}, \quad p=2,3,4, \\
u(0, x)=Q(x)
\end{array}\right.
$$

where $a$ is an unknown control. Our first result states that any gKdV soliton is approximate null controllability for sufficiently large time.

THEOREM 1.1. Any gKdV soliton is approximate null-controllable in large time. More precisely, fix $\delta_{0}>0$ small. There is $\delta_{1}=\delta_{1}\left(\delta_{0}\right)>0$ small such that for all $0<\delta<\delta_{1}$, the following holds. There exist a time $T=T_{\delta}>0$, and a smooth control $a=a_{\delta}(t, \cdot) \in L^{\infty}(\mathbb{R}) \cap L^{2}(\mathbb{R})$, defined in $[0, T]$, such that the unique solution $u=u_{\delta}(t)$ of (1.1) in $C\left([0, T], H^{1}(\mathbb{R})\right)$, with initial condition $u_{0}(x)=Q(x)$, satisfies

$$
\|u(T)\|_{H^{1}(\mathbb{R})} \leq \delta .
$$

Finally, one has $T \sim \delta^{-2\left(1+\delta_{0}\right)}$ and $\sup _{t}\left\|a_{\delta}(t)\right\|_{L^{2} \cap L^{\infty}} \lesssim \delta^{2}$.

As far as we know, this is the first (partial) result on controllability of solitons in unbounded domains, where dispersion plays a key role in the dynamics. Previous results are related to the study of the ground state of the linear and the nonlinear problem in a finite interval, see e.g. the works of Lange and Teismann [16, Beauchard and Mirrahimi [2, Mirrahimi [27, or the control of a quantum particle under the action of a well shaped potential, obeying the linear Schrödinger equation in a bounded interval (Beauchard-Coron [3]), and Crépeau [9] in the KdV case. In this paper we study a nonlinear object instead; localized solitons are present due the non compact character of the domain, and the strength of the nonlinearity.

It turns out that Theorem 1.1 is consequence of the following deeper result, a large time approximate controllability of the initial soliton $Q$ of scaling one, to any final scaling $c_{f}>0, c_{f} \neq 1$ (the case $c_{f}=1$ is trivial). As previously stated, we pick any $\delta_{0}>0$ small, but fixed.

Theorem 1.2. Let $c_{f}>0, c_{f} \neq 1$. There exists $\varepsilon_{0}\left(\delta_{0}, c_{f}\right)>0$ such that, for all $0<\varepsilon<\varepsilon_{0}$ the following holds. There exist a time $T=T_{\varepsilon}>0$, a smooth in time and space control $a=a_{\varepsilon}(t, \cdot) \in L^{\infty}(\mathbb{R}) \cap L^{2}(\mathbb{R})$ and a smooth translation parameter $\rho(t)$, both defined in $[0, T]$, and such that the unique solution $u=u_{\varepsilon}(t)$ of (1.1) in $C\left([0, T], H^{1}(\mathbb{R})\right)$, with initial condition $u_{0}(x)=Q(x)$, satisfies

$$
\left\|u(T)-Q_{c_{f}}(\cdot-\rho(T))\right\|_{H^{1}(\mathbb{R})}+\left|\rho^{\prime}(T)-c_{f}\right| \lesssim \sqrt{\varepsilon} .
$$

Finally, one has $T \sim \varepsilon^{-1-\delta_{0}}$ and $\sup _{t}\left\|a_{\varepsilon}(t)\right\|_{L^{2} \cap L^{\infty}} \lesssim \varepsilon$.

Proof. [Proof of Theorem 1.1, assuming Theorem 1.2, The proof of Theorem 1.1 follows from Theorem 1.2, and the subcritical character of solitons for $p<5$. Indeed, just take any $0<c_{f} \leq \frac{1}{100} \delta^{\frac{4(p-1)}{5-p}}$ and $\varepsilon_{0} \lesssim \delta^{2}$ in Theorem 1.2. From (1.2) we have, after integration and rescaling,

$$
\left\|Q_{c_{f}}\right\|_{H^{1}(\mathbb{R})} \sim c_{f}^{\frac{5-p}{4(p-1)}}<\frac{1}{2} \delta .
$$


Therefore, using (1.9),

$$
\|u(T)\|_{H^{1}(\mathbb{R})} \lesssim \sqrt{\varepsilon}+\left\|Q_{c_{f}}(\cdot-\rho(T))\right\|_{H^{1}(\mathbb{R})} \leq \frac{1}{2} \delta+\sqrt{\varepsilon} \leq \delta .
$$

Note that Theorem 1.1 holds even without destroying the soliton structure. $\square$

Some comments about Theorem 1.2

REMARK 1.1. First of all, we point out that the control a is not compactly supported, but it satisfies the following properties (see Section 0 and (2.13) for more details):

1. It is exponentially decreasing in any moving region far away form the soliton (in other words, it moves with the soliton);

2. It has slow variation in space, which actually explains the large time needed in order to drive the dynamics.

REMARK 1.2. Second, as for the final position and time of control $T$ are concerned, we obtain estimates of the following orders: for any $\delta_{0}>0$ small but fixed,

$$
T \sim \varepsilon^{-1-\delta_{0}}, \quad\left|\rho(T)-c_{f} T\right| \lesssim \varepsilon^{-1 / 2-\delta_{0}},
$$

although the relative error in the last estimate is $O\left(\varepsilon^{1 / 2}\right)$. The relative weakness in $\varepsilon$ of the last estimates and the bound (1.9) is mainly due to the emergence of dispersive tails behind the soliton solution as the control acts; this phenomenon has been observed in several interaction problems involving gKdV equations, starting from the formal arguments in [13, 15, 10], and the more rigorous treatment given in [23, 24, 28, [29, 11, 12]. This phenomenon does not appear in the case of nonlinear Schrödinger equations, where one expects better estimates (see e.g. [29]). Heuristically speaking, the lack of control on the position, compared to the extremely accurate control on the velocity, could be associated to a form of uncertainty principle for solitons, regarded this time as almost point particles.

REMARK 1.3. A necessary condition to obtain an estimate as in (1.9) is the lack of conserved quantities (see Proposition 2.2). Indeed, it is not difficult to see that the soliton at time $T$ has lost or gained, depending on the sign of a and $c_{f}$, a nontrivial $O(1)$ amount of mass (1.5). A similar study can be applied to the case of the energy (1.6), with similar conclusions. It is important to stress that, since solitons are stable under small $H^{1}$ perturbations [1, 4, 40] and the equation is not integrable unless $p=2$ or $p=3$, it is expected that the result above only holds if we introduce a sufficiently slowly varying potential.

On the other hand, problem (1.1) can be also regarded as a stabilization problem. In that sense, the recent literature concerns with the decay of solutions posed in a bounded interval [35, 25, 5, 17, or the half line by Linares and Pazoto [19, 20, 30, and numerical schemes for the critical case $p=5$ (Pazoto et. al. [31]). As for the decreasing mass case, and the approximate null controllability result stated in Theorem 1.1 we have the following additional approximate stabilization result, without destroying the soliton:

Corollary 1.3. Under the assumptions of Theorem 1.1, there exist $C, \mu_{0}>0$, independent of $\delta \in\left(0, \delta_{1}\right)$ such that, for all $t \in[0, T]$

$$
\|u(t)\|_{H^{1}(\mathbb{R})} \leq C\left(\delta+e^{-\mu_{0} \delta^{2} t}\right)\|Q\|_{H^{1}(\mathbb{R})}, \quad p=2,3,4 .
$$


REMARK 1.4. Finally, some words about the corresponding exact controllability problem. A nice exact controllability result could be obtained if we were able to prove e.g. exact null controllability of small solitons, and then combining Theorems 1.1] and 1.9 in the standard way. However, based on some results about inelasticity of the dynamics for slightly perturbed solitons (cf. [28, 29]), we believe that in our model, and in more general situations, solitons are never exactly controllable, even in infinite time.

Indeed, note that if (1.8) is exactly controllable to zero in finite time, say $u(T)=0$ for some bounded, smooth control $a(t, x)$, then using the reversibility in time of the equation, we have that $v(t, x):=u(T-t,-x)$ satisfies a slightly different equation,

$$
v_{t}+\left(v_{x x}+v^{p}\right)_{x}=b(t, x) v, \quad v(0)=0,
$$

for the potential $b(t, x):=a(T-t,-x)$. It turns out that, under standard assumptions on the solvability of the Cauchy problem associated to $v$, the unique solution to the above problem is the identically zero solution, a contradiction. I thank Sylvain Ervedoza for this remark.

1.1. About the proofs. Our proofs do not involve the usual methods employed in control theory, requiring e.g. the study of the linear problem, unique continuation properties and/or Carleman estimates. In order to study genuine nonlinear objects such as solitons, we need different dispersive methods. In particular, a suitable global well-posedness theory in the energy space for solutions of (1.1) in the real line requires modifications on the arguments of the fundamental work by Kenig, Ponce and Vega [14, in order to deal with the unbounded domain case. Second, our control is explicitly constructed, with the following properties: $(i)$ it has a slowly varying character, determined by the parameter $\varepsilon$; (ii) it is localized in a moving region of size $O(1)$, and is of strength $O(\varepsilon)$ (but it is not compactly supported), and (iii) the corresponding slowly varying part induces on the soliton parameters a finite dimensional dynamical system which governs the whole dynamics. Concerning the time of control, since the dynamics is slowly varying, the time of interaction is $O\left(\varepsilon^{-1}\right)$ at least; a large control introduced in a smaller window of time could destroy the soliton.

The second step of the proof is the following: since the introduction of the control induces on the soliton the action of an external potential, we can think such an interaction as a slowly varying collision between both objects. In Section 3 , we construct an explicit approximate solution which describes the interaction, up to certain order of accuracy in $\varepsilon$. This solution $\tilde{u}(t)$ has the form

$$
u(t, x) \sim Q_{c(t)}(x-\rho(t))+\varepsilon A(t, x-\rho(t)),
$$

where $\varepsilon>0$ is a small, artificially introduced parameter, and $(c(t), \rho(t))$ are suitable scaling and translation parameters, depending on time. The parameters follow a suitable approximate finite-dimensional, slowly varying in time dynamics, determined by the action of the control, described as follows:

$$
c^{\prime}(t) \sim a_{x}(t, \varepsilon \rho(t)), \quad \rho^{\prime}(t) \sim c(t)+O(\varepsilon),
$$

where $a$ is the control introduced in (2.13). We choose carefully $a$ such that the evolution of this system leads to the desired final velocity, at time $T \sim \varepsilon^{-1-\delta_{0}}$,

$$
c(T) \sim c_{f}, \quad \rho(T) \sim c_{f} T+o(T),
$$


however, a better control on the position has escaped to us.

Concerning the function $A$, it corresponds to a first order correction term with support of size $O\left(\varepsilon^{-1}\right)$ in the variable $x-\rho(t)$ (the soliton variable), and $L^{\infty}$-norm of order $O\left(e^{-\gamma_{0} \varepsilon|\rho(t)|}\right)$, for some constant $\gamma_{0}>0$. Therefore, $A$ is a phantom term that disappears after the interaction, but which allows to improve the accuracy of the approximate solution. Finding $A$ is an absolutely necessary condition, otherwise a bound like (1.9) is highly unlikely. Additionally, $A$ is in principle only bounded, but not localized 2 therefore we introduce a suitable cut-off function to recover a finite mass solution. The error associated to this approximation is measured in terms of the $L_{t}^{\infty} H_{x}^{1}$ norm, and it has to be small enough in order to take into account the large time of interaction. In our case, we are able to prove that during the whole interaction, one has

$$
\text { error } \sim \varepsilon^{3 / 2} e^{-\gamma_{0} \varepsilon|\rho(t)|}, \quad t \in[0, T],
$$

(see (3.47)), therefore the propagation of this error during a time interval of order $\sim T$ formally leads to the bound $O(\sqrt{\varepsilon})$ in Theorem 1.2. We remark that this method has been recently applied, in a different context, to several interaction problem, notably the two-soliton collision by Y. Martel and F. Merle [23, 24, and the interaction of solitons with a potential 28, 29. See also 32 for a related soliton-potential problem in a different context, in the easier case of the cubic nonlinearity, and for which the term $A$ is not needed.

The third step of the proof is the following. In order to control the dynamics of the error terms, we introduce a suitable Lyapunov functional (Section 4), adapted this time to the genuine nonlinear dynamics of the problem (see e.g. 23). This functional has very small variation in time, provided we control the size of some time dependent parameters of the soliton solution. We avoid that problem by using sharp virial estimates, in the spirit of [22]. After this point, we can close the main argument by proving rigorously that the error terms can be assured to be smaller than $O(\sqrt{\varepsilon})$, during the whole interaction region.

The final step of the proof is a rigorous analysis of the parameters $(c(t), \rho(t))$ of the soliton solution, in order to recover (1.11). We prove that at time $t=T$, the solution has the desired behavior, up to an error of $O(\sqrt{\varepsilon})$, finishing the proof of Theorem 1.2. Finally, the proof of Corollary 1.3 follows after a detailed study of the scaling parameter $c(t)$.

The weakness of our approach is precisely the approximate character of the controllability property, and the large time needed to reach an approximate final state. We believe that our results can be improved by adapting to this case, the standard and complex machinery of control theory. Additionally, we believe that the moving profile of the support can be chosen to be compactly supported.

We point out that in order to describe the dynamics in a time of order $O(1)$, one formally needs a large control; in particular, it should be unbounded in space (more precisely, linearly growing in space). However, even the local in time Cauchy problem for such perturbations becomes a very difficult problem.

Finally, some words about the organization of this paper. In Section 2 , we introduce the explicit control system, the finite dimensional dynamical system and the

\footnotetext{
${ }^{2}$ In principle, $A$ models a dispersive tail behind the soliton solution.
} 
corresponding local and global well-posedness theory. In Section 3 we construct an approximate solution to a given order of accuracy. We continue this process up to the moment when we find an infinite mass correction term, which is up to date the best mathematical description of the dispersive tail behind the soliton, originated by the application of the control. Section 4 is devoted to the introduction of a Lyapunov function, modulation theory and a key virial identity in order to control the dynamics of the oscillatory terms. Finally, in Section 5 we prove the main theorem.

Notation. Along this paper we use the convention $A \lesssim B$ if and only if there exists $K>0$, independent of $\varepsilon$, such that $A \leq K B$. Additionally, $\gamma$ and $K^{*}$ will denote special positive constants, still independent of $\varepsilon$, to be worried about. Finally, $\mathcal{S}(\mathbb{R})$ denotes the Schwartz's class on $\mathbb{R}$.

Acknowledgments. I would like to thank the referees for their useful and constructive critiscisms. I also thank Gunther Uhlmann and Axel Osses for their kind invitation to the PASI-CIPPDE 2012, Inverse Problems and PDE Control, held in Santiago-Chile, and where this project was originally conceived. Finally I'm grateful of Eduardo Cerpa and Sylvain Ervedoza, for many useful comments and suggestions to a first draft of this paper.

2. First ingredients. Let $p=2,3$ or 4 . Given any $c_{f}>0$ fixed and $Q$ be the soliton defined in (1.3), we define the quantities

$$
\begin{gathered}
\lambda_{p}:=\frac{4(p-1)}{5-p} \frac{\int_{\mathbb{R}} Q^{3}}{\int_{\mathbb{R}} Q^{2}}>0, \\
a_{\infty}:=-\frac{1}{\lambda_{2}} \log c_{f}, \quad p=2, \quad a_{\infty}:=\frac{(p-1)}{\lambda_{p}(p-2)}\left(1-c_{f}^{\frac{p-2}{p-1}}\right), \quad p=3,4 .
\end{gathered}
$$

Note that, as expected, for every $p$ the value of $a_{\infty}\left(c_{f}\right)$ tends to zero as $c_{f}$ approaches the trivial case $c_{f}=1$ (i.e. no control is needed).

We introduce now the control $a(t, x)$. Given any $\varepsilon>0$ small, we consider a smooth function $a_{0}$ satisfying the following properties (recall that $A \lesssim B$ means that there is $C>0$ such that $A \leq C B$ )

$$
\left\{\begin{array}{l}
a_{0} \in C^{3}(\mathbb{R}) \cap L^{\infty}(\mathbb{R}), \\
\left|a_{0}(x)\right| \lesssim e^{\gamma_{0} x}, \text { for } x \leq-1, \quad\left|a_{\infty}-a_{0}(x)\right| \lesssim e^{-\gamma_{0} x} \text { for } x \geq 1 \\
\left|a_{0}^{(k)}(x)\right| \lesssim e^{-\gamma_{0}|x|}, \quad x \in \mathbb{R}, k=1,2,3 \\
a_{0}^{\prime}(x)>0 \text { if } a_{\infty}>0, \quad a_{0}^{\prime}(x)<0 \quad \text { if } a_{\infty}<0
\end{array}\right.
$$

for a fixed, positive constant $\gamma_{0}$. Note that with this choice,

$$
\left\|a_{0}\right\|_{\infty}=\left|a_{\infty}\right| .
$$

Let $0<c_{m}:=\frac{1}{2} \min \left\{c_{f}, 1\right\}, c_{M}:=2 \max \left\{1, c_{f}\right\}$, and $\left(c_{0}(t), \rho_{0}(t)\right) \in \mathbb{R}_{+} \times \mathbb{R}$ be a set of $C^{1}$ parameters defined in $\{t \geq 0\}$, with the following uniform, a-priori constraints

$$
0<c_{m} \leq c_{0}(t), \rho_{0}^{\prime}(t) \leq c_{M}
$$


More precisely, consider $t \geq 0$ and $\left(c_{0}(t), \rho_{0}(t)\right) \in \mathbb{R}_{+} \times \mathbb{R}$ be the unique solution of the nonlinear ODE system

$$
\left\{\begin{array}{l}
c_{0}^{\prime}(t)=\varepsilon f_{1}^{0}\left(c_{0}(t), \rho_{0}(t)\right), \quad c_{0}(0)=1, \\
\rho_{0}^{\prime}(t)=c_{0}(t), \quad \rho_{0}(0)=-\varepsilon^{-1-\delta_{0}},
\end{array}\right.
$$

where $f_{1}^{0}$ is defined as follows (cf. (2.1))

$$
f_{1}^{0}(c, \rho):=-\lambda_{p} a_{0}^{\prime}(\varepsilon \rho) c^{\frac{p}{p-1}}
$$

and $\delta_{0}>0$ is the small parameter of Theorems 1.1 and 1.2 Additionally, we will need the following function

$$
f_{2}^{0}(c, \rho):=\mu_{p} a_{0}^{\prime}(\varepsilon \rho) c^{\frac{2(5-2 p)}{7-3 p}},
$$

for some $\mu_{p} \in \mathbb{R}$, with $\mu_{3}=0$ (note that $\frac{2(5-2 p)}{7-3 p}>0$ for $p=2,3,4$ ).

Lemma 2.1. There exists a unique solution $\left(c_{0}(t), \rho_{0}(t)\right) \in \mathbb{R}_{+} \times \mathbb{R}$ of (2.6), defined for all $t \geq 0$. Moreover, we have

$$
\lim _{t \rightarrow+\infty} c_{0}(t)=c_{f}\left(1+O\left(\varepsilon^{10}\right)\right), \quad \lim _{t \rightarrow+\infty} \rho_{0}(t)=+\infty
$$

and

$$
c_{m} \leq c_{0}(t) \leq c_{M}
$$

for $\varepsilon$ small enough.

Proof. The existence of a unique local solution to (2.6) is a direct consequence of the Cauchy-Lipschitz-Picard theorem. The global character of the solution is directly determined by the boundedness of $a_{0}$.

Let us prove (2.9) and (2.10). First of all, note that $\left(c_{0}(t), \rho_{0}(t)\right) \equiv(0$, constant) is a constant solution of (2.6), without considering the initial conditions. Therefore, we have $c_{0}(t)>0$ for all $t \geq 0$. On the other hand, from the first equation in (2.6),

$$
c_{0}^{-\frac{1}{p-1}}(t) c_{0}^{\prime}(t)=-\varepsilon \lambda_{p} a_{0}^{\prime}\left(\varepsilon \rho_{0}(t)\right) c_{0}(t)=-\varepsilon \lambda_{p} a_{0}^{\prime}\left(\varepsilon \rho_{0}(t)\right) \rho_{0}^{\prime}(t) .
$$

Hence, if $p=2$,

$$
\log c_{0}(t)=-\lambda_{2}\left[a_{0}\left(\varepsilon \rho_{0}(t)\right)-a_{0}\left(-\varepsilon^{-\delta_{0}}\right)\right] .
$$

from which we obtain for $\varepsilon$ small, using (2.3),

$$
c_{0}(t)=e^{-\lambda_{2} a_{0}\left(\varepsilon \rho_{0}(t)\right)}\left(1+O\left(\varepsilon^{10}\right)\right), \quad p=2,
$$

with the term $O\left(\varepsilon^{10}\right)$ independent of time. Similarly, if $p=3$ or 4 ,

$$
c_{0}(t)=\left[1-\lambda_{p} \frac{(p-2)}{p-1} a_{0}\left(\varepsilon \rho_{0}(t)\right)\right]^{\frac{p-1}{p-2}}\left(1+O\left(\varepsilon^{10}\right)\right) .
$$

Note that from (2.11)-(2.12), (2.2) and (2.3), $c_{0}(t)$ satisfies the bounds

$$
0<c_{m}=\frac{1}{2} \min \left\{c_{f}, 1\right\} \leq c_{0}(t) \leq 2 \max \left\{c_{f}, 1\right\}=c_{M}
$$


This shows (2.10). We conclude that for $\varepsilon>0$ small, $\rho_{0}(t)$ is increasing and $\rho_{0}(t)-$ $\rho_{0}(0) \geq c_{m} t$, which implies that $\lim _{\infty} \rho_{0}=+\infty$. Moreover, from (2.3),

$$
\lim _{+\infty} a\left(\varepsilon \rho_{0}(t)\right)=a_{\infty}
$$

Therefore

$$
\lim _{+\infty} c_{0}(t)=e^{-\lambda_{2} a_{\infty}}\left(1+O\left(\varepsilon^{10}\right)\right)=c_{f}\left(1+O\left(\varepsilon^{10}\right)\right), \quad p=2,
$$

and similarly for $p=3,4$. This proves (2.9).

Finally, define

$$
a(t, x):=-\varepsilon a_{0}^{\prime}(\varepsilon x) Q_{c_{0}(t)}\left(x-\rho_{0}(t)\right),
$$

where $Q_{c}$ is the solution of (1.4) and $\left(c_{0}(t), \rho_{0}(t)\right)$ is the solution of (2.6). Let us remark that this control takes into account important information of the soliton itself, namely the approximate scaling $c_{0}(t)$ and position $\rho_{0}(t)$, and it is in some sense of nonlinear character. In terms of numerical applications, these two parameters can be easily described by solving the ODE (2.6). The non stationary character of this control will become essential in the proof.

It is not difficult to check that this control satisfies the following space-time bounds

$$
\|a\|_{L^{\infty}([0, \infty) \times \mathbb{R})}+\left\|a_{x x}\right\|_{L^{\infty}([0, \infty) \times \mathbb{R})} \lesssim \varepsilon .
$$

Under these estimates, we claim that the Cauchy problem associated to (1.1) is locally well-posed in a subspace of $H^{1}(\mathbb{R})$.

Proposition 2.2. Under the assumptions (2.3), (2.5), (2.6) and (2.13), the initial value problem (1.8) is locally well-posed in $H^{1}(\mathbb{R})$. Moreover, the mass $M[u](t)$ and energy $E[u](t)$ defined in (1.5) and (1.6) satisfy the relations

$$
\partial_{t} M[u](t)=\int_{\mathbb{R}} a(t, x) u^{2}, \quad \partial_{t} E[u](t)=-\frac{1}{2} \int_{\mathbb{R}} a_{x x} u^{2}-\int_{\mathbb{R}} a u^{p+1}+\int_{\mathbb{R}} a u_{x}^{2} .
$$

REMARK 2.1. Later we will prove that our solution is well-defined, for all $t \leq$ $T \sim \varepsilon^{-1-\delta_{0}}$, as a consequence of the stability property (1.9).

Proof. This result is classical, see e.g. Merle-Vega [26 in the case where $p=3$ (the so called $\mathrm{mKdV}$ equation) and the nonlinearity $u^{p}$ has the opposite sign. For the sake of completeness, we sketch the main details. We use the machinery developed by Kenig, Ponce and Vega [14 to prove local well-posedness for gKdV in low regularity Sobolev spaces. Since we only need an $H^{1}$ local theory, our proof will be simpler than the original one.

Recall that we want to solve

$$
u_{t}+u_{x x x}=-\left[p u^{p-1} u_{x}+a(t, x) u\right], \quad u(0)=u_{0} \in H^{1}(\mathbb{R}) .
$$

If we denote by $e^{-t \partial_{x}^{3}}$ the free Airy propagator, we have to solve fixed point problem

$$
\begin{aligned}
u(t) & =\mathcal{T}[u](t):=e^{-t \partial_{x}^{3}} u_{0}-\int_{0}^{t} e^{-(t-s) \partial_{x}^{3}}\left[p u^{p-1} u_{x}+a u\right](s) d s \\
& :=\mathcal{T}_{0}\left[u_{0}\right](t)+\mathcal{T}_{1}[u](t) .
\end{aligned}
$$


Note that, since we have chosen $(c, \rho)$ following (2.5),

$$
\int_{\mathbb{R}} \sup _{0 \leq t \leq 1} a^{2} \lesssim \varepsilon^{2} \int_{\mathbb{R}} a_{0}^{\prime 2}(\varepsilon x) d x \lesssim \varepsilon, \quad \sup _{0 \leq t \leq 1}\|a\|_{L_{x}^{\infty}(\mathbb{R})} \lesssim \varepsilon .
$$

Using [14, Theorem 3.5] and the maximal function estimate [14, (3.9)], we have, for any $S \in(0,1)$,

$$
\begin{aligned}
\sup _{0 \leq t \leq S}\left\|\partial_{x} \mathcal{T}_{1}[u]\right\|_{L^{2}(\mathbb{R})} & \leq \int_{\mathbb{R}_{x}}\left\|p u^{p-1} u_{x}+a u\right\|_{L^{2}(0 \leq t \leq S)} d x \\
& \lesssim\left(\int_{\mathbb{R}} \sup _{0 \leq t \leq S}|u|^{2 p-2} d x\right)^{1 / 2}\left\|u_{x}\right\|_{L^{2}([0, S] \times \mathbb{R})} \\
& \quad+\left(\int_{\mathbb{R}} \sup _{0 \leq t \leq S} a^{2} d x\right)^{1 / 2}\|u\|_{L^{2}([0, S] \times \mathbb{R})} \\
& \lesssim S^{1 / 2}\|u\|_{L^{\infty}\left((0, S) ; H^{1}(\mathbb{R})\right)}^{p}+\sqrt{\varepsilon} S^{1 / 2}\|u\|_{L^{\infty}\left((0, S) ; L^{2}(\mathbb{R})\right)} .
\end{aligned}
$$

On the other hand,

$$
\begin{aligned}
\left\|\mathcal{T}_{1}[u]\right\|_{L^{2}\left(\mathbb{R}_{x}\right)} \lesssim & \int_{0}^{S}\left[\left\|u^{p-1} u_{x}\right\|_{L^{2}\left(\mathbb{R}_{x}\right)}+\|a u\|_{L^{2}\left(\mathbb{R}_{x}\right)}\right] d t \\
\lesssim & S \sup _{0 \leq t \leq S}\left(\int_{0}^{S}\|u\|_{L^{\infty}\left(\mathbb{R}_{x}\right)}^{p-1}\right)^{1 / 2}\left\|u_{x}\right\|_{L^{2}\left(\mathbb{R}_{x}\right)} \\
& +S \sup _{0 \leq t \leq S}\|a\|_{L^{\infty}\left(\mathbb{R}_{x}\right)}\|u\|_{L^{2}(\mathbb{R})} .
\end{aligned}
$$

It is not difficult to check that these estimates give that, for $S$ small, $\mathcal{T}$ maps a ball of $H^{1}$ into itself. The contraction follows in a similar way.

Let $T_{0}>0$ be the maximal time of existence of a solution $u(t)$. It is not difficult to check that the mass and energy (1.5)-(1.6) satisfy, for all $t \in\left[0, T_{0}\right)$,

$$
\partial_{t} M[u](t)=\int_{\mathbb{R}} a(t, x) u^{2} \lesssim \varepsilon M[u](t),
$$

therefore $M[u](t) \lesssim e^{C \varepsilon t}$. On the other hand, the energy (1.6) satisfies the relation

$$
\partial_{t} E[u](t)=-\frac{1}{2} \int_{\mathbb{R}} a_{x x}(t, x) u^{2}-\int_{\mathbb{R}} a(t, x) u^{p+1}+\int_{\mathbb{R}} a(t, x) u_{x}^{2}
$$

3. Approximate solution. Given any $\varepsilon>0$ and $\delta_{0}>0$ small, we introduce the time of interaction

$$
T:=\min \left\{T_{0}, \varepsilon^{-1-\delta_{0}}\right\},
$$

where $T_{0}>0$ is the maximal time of existence of the solution $u(t)$ with initial condition $Q(x)$.

Let $t \in[0, T]$. In what follows, we fix a couple of dynamical parameters $(c(t), \rho(t))$, a perturbation of the couple $\left(c_{0}(t), \rho_{0}(t)\right)$, and satisfying the same estimates (2.5) in the same subinterval of $\{t \geq 0\}$. Additionally, we will assume that

$$
\left|c(t)-c_{0}(t)\right|+\left|\rho(t)-\rho_{0}(t)\right| \leq \varepsilon^{1 / 2-\delta_{0}},
$$


for all $t \in[0, T]$.

Now we define the modulated soliton solution as follows. Let

$$
y:=x-\rho(t), \quad R(t, x):=Q_{c(t)}(y) .
$$

Finally, we introduce the approximate solution

$$
\tilde{u}(t, x):=R(t, x)+w(t, x), \quad w(t, x):=\varepsilon d(t) A_{c(t)}(t, y), \quad d(t):=a_{0}^{\prime}(\varepsilon \rho(t)) ;
$$

for some $L^{\infty}(\mathbb{R})$ function $A_{c}(t, \cdot)$, to be introduced later. In order to simplify some computations, we will assume that for $c_{m} \leq c(t) \leq c_{M}$ and $t$ fixed, $A_{c(t)}(t, \cdot)$ satisfies the estimates

$$
A_{c}^{\prime}(t, \cdot) \in L^{2}(\mathbb{R}), \quad \partial_{c} A_{c}(t, \cdot) \in L^{\infty}(\mathbb{R}),
$$

that will be verified below. Finally, we define the scaling operator

$$
\Lambda Q_{c}(y):=\left.\partial_{c^{\prime}} Q_{c^{\prime}}\right|_{c^{\prime}=c}(y)=\frac{1}{c}\left[\frac{1}{p-1} Q_{c}(y)+\frac{1}{2} y Q_{c}^{\prime}(y)\right] \in \mathcal{S}(\mathbb{R}) .
$$

We want to measure the size of the error induced by inserting $\tilde{u}$ as defined in (3.3) in the equation (1.8)-(2.13). Let

$$
S[\tilde{u}](t, x):=\tilde{u}_{t}+\left(\tilde{u}_{x x}+\tilde{u}^{p}\right)_{x}+\varepsilon a_{0}^{\prime}(\varepsilon x) Q_{c_{0}(t)}\left(x-\rho_{0}(t)\right) \tilde{u} .
$$

From (3.2) we have

$$
\begin{aligned}
Q_{c_{0}(t)}\left(x-\rho_{0}(t)\right)= & Q_{c(t)}(x-\rho(t))+\Lambda Q_{c(t)}(x-\rho(t))\left(c_{0}(t)-c(t)\right) \\
& +Q_{c(t)}^{\prime}(x-\rho(t))\left(\rho_{0}(t)-\rho(t)\right)+O_{H^{1}(\mathbb{R})}\left(\varepsilon^{1-2 \delta_{0}}\right) .
\end{aligned}
$$

Our first result is the following

Proposition 3.1. Let $(c, \rho)$ be satisfying (2.5) and (3.2). There exists a function $A_{c} \in L^{\infty}(\mathbb{R})$ such that $\tilde{u}$, defined in (3.3), satisfie 3

$$
S[\tilde{u}](t, x)=\left(c^{\prime}(t)-\varepsilon f_{1}(t)\right) \partial_{c} \tilde{u}-\left(\rho^{\prime}(t)-c(t)-\varepsilon f_{2}(t)\right) \partial_{y} \tilde{u}+\tilde{S}[\tilde{u}],
$$

where $f_{1}(t)=f_{1}(c(t), \rho(t))$ and $f_{2}(t)=f_{2}(c(t), \rho(t))$ are given by

$$
\begin{aligned}
f_{1}(c(t), \rho(t))= & f_{1}^{0}(c(t), \rho(t))+O\left(a_{0}^{\prime}(\varepsilon \rho(t))\left|c_{0}(t)-c(t)\right|\right), \\
f_{2}(c(t), \rho(t))= & f_{2}^{0}(c(t), \rho(t)) \\
& +O\left(a_{0}^{\prime}(\varepsilon \rho(t))\left(\left|\rho_{0}(t)-\rho(t)\right|+\left|c_{0}(t)-c(t)\right|\right)\right),
\end{aligned}
$$

with $f_{1}^{0}(t), f_{2}^{0}(t)$ defined in 2.7)-(2.8). Moreover,

$$
\|\tilde{S}[\tilde{u}](t)\|_{H^{1}\left(y>-\frac{2}{\varepsilon}\right)} \lesssim \varepsilon^{3 / 2} e^{-\gamma \varepsilon|\rho(t)|}+\varepsilon\left|c(t)-c_{0}(t)\right| e^{-\varepsilon \gamma|\rho(t)|}+\varepsilon^{3},
$$

and

$$
\left|\int_{\mathbb{R}} Q_{c} \tilde{S}[\tilde{u}]\right|+\left|\int_{\mathbb{R}} y Q_{c} \tilde{S}[\tilde{u}]\right| \lesssim \varepsilon^{2} e^{-\varepsilon \gamma|\rho(t)|}+\varepsilon\left|c(t)-c_{0}(t)\right| e^{-\varepsilon \gamma|\rho(t)|}+\varepsilon^{3} .
$$

\footnotetext{
${ }^{3}$ The first two terms in 3.8 are often referred as the finite-dimensional dynamical system associated to the soliton dynamics.
} 
Proof. We follow the strategy described in 29. Suppose that the parameters $(c(t), \rho(t))$ satisfy (2.5) and (3.2). From (3.6), we have

$$
S[\tilde{u}]=\mathbf{I}+\mathbf{I I}+\mathbf{I I I},
$$

where (we omit the dependence on $t$ and $x$ if it is not necessary)

$$
\mathbf{I}:=S[R], \quad \mathbf{I I}=\mathbf{I I}(w):=w_{t}+\left(w_{x x}+p R^{p-1} w\right)_{x}+\varepsilon a_{0}^{\prime}(\varepsilon x) Q_{c_{0}}\left(x-\rho_{0}\right) w,
$$

and for $p=2,3$ or 4 ,

$$
\text { III }:=\left\{(R+w)^{p}-R^{p}-p R^{p-1} w\right\}_{x} .
$$

Recall that $w$ is given by (3.3). In the next results, we expand the terms in (3.13). Note that $R(t, x)=Q_{c(t)}(y)$ and $y=x-\rho(t)$.

LEMMA 3.2.

$$
\mathbf{I}=F_{0}^{\mathbf{I}}(t, y)+\varepsilon F_{1}^{\mathbf{I}}(t, y)+\varepsilon^{2\left(1-\delta_{0}\right)} F_{c}^{\mathbf{I}}(t, y),
$$

where

$$
F_{0}^{\mathbf{I}}(t, y):=\left(c^{\prime}(t)-\varepsilon f_{1}(t)\right) \partial_{c} R(t)-\left(\rho^{\prime}(t)-c(t)-\varepsilon f_{2}(t)\right) \partial_{x} R(t),
$$

$f_{1}(t)$ and $f_{2}(t)$ are given by (3.9)-(3.10), and

$$
\begin{aligned}
F_{1}^{\mathbf{I}}(t ; y):= & f_{1}(t) \Lambda Q_{c}(y)+a_{0}^{\prime}(\varepsilon \rho) Q_{c}^{2}(y)-f_{2}(t) Q_{c}^{\prime}(y) \\
& +\left(c_{0}-c\right) a_{0}^{\prime}(\varepsilon \rho) \Lambda Q_{c} Q_{c}(y)+\left(\rho_{0}-\rho\right) a_{0}^{\prime}(\varepsilon \rho) Q_{c}^{\prime} Q_{c}(y) .
\end{aligned}
$$

Finally, for all $t \in[0, T]$, one has $\left\|F_{c}^{\mathbf{I}}(t, \cdot)\right\|_{H^{1}(\mathbb{R})} \lesssim e^{-\varepsilon \gamma|\rho(t)|}+\varepsilon$.

Proof. [Proof of Lemma 3.2] We have

$$
\begin{aligned}
\mathbf{I} & =R_{t}+\left(R_{x x}+R^{p}\right)_{x}+\varepsilon a_{0}^{\prime}(\varepsilon x) Q_{c_{0}} Q_{c} \\
& =c^{\prime} \Lambda Q_{c}-\rho^{\prime} Q_{c}^{\prime}+Q_{c}^{(3)}+\left(Q_{c}^{p}\right)^{\prime}+\varepsilon a_{0}^{\prime}(\varepsilon x) Q_{c_{0}} Q_{c} .
\end{aligned}
$$

On the other hand, note that via a Taylor expansion,

$$
a_{0}^{\prime}(\varepsilon x) Q_{c}=a_{0}^{\prime}(\varepsilon \rho) Q_{c}+\varepsilon a_{0}^{\prime \prime}(\varepsilon \rho) y Q_{c}+O_{H^{1}(\mathbb{R})}\left(\varepsilon^{2}\right) .
$$

Therefore, using the equation satisfied by $Q_{c}$, namely, $Q_{c}^{\prime \prime}-c Q_{c}+Q_{c}^{p}=0$, (3.7) and (3.2), we have

$$
\begin{aligned}
\mathbf{I}= & c^{\prime} \Lambda Q_{c}-\left(\rho^{\prime}-c\right) Q_{c}^{\prime}+Q_{c}^{(3)}-c Q_{c}^{\prime}+\left(Q_{c}^{p}\right)^{\prime}+\varepsilon a_{0}^{\prime} Q_{c_{0}} Q_{c} \\
& +\varepsilon^{2} a_{0}^{\prime \prime} y Q_{c_{0}} Q_{c}+O_{H^{1}(\mathbb{R})}\left(\varepsilon^{3}\right) \\
= & \left(c^{\prime}-\varepsilon f_{1}\right) \Lambda Q_{c}-\left(\rho^{\prime}-c-\varepsilon f_{2}\right) Q_{c}^{\prime} \\
& +\varepsilon\left[f_{1} \Lambda Q_{c}+a_{0}^{\prime} Q_{c}^{2}-f_{2} Q_{c}^{\prime}+\left(c_{0}-c\right) a_{0}^{\prime} Q_{c} \Lambda Q_{c}+\left(\rho_{0}-\rho\right) a_{0}^{\prime} Q_{c}^{\prime} Q_{c}\right] \\
& +\varepsilon^{2\left(1-\delta_{0}\right)} F_{c}^{\mathbf{I}}(t, y),
\end{aligned}
$$

with $F_{c}^{\mathbf{I}}(t, \cdot) \in \mathcal{S}(\mathbb{R})$ and $\left\|F_{c}^{\mathbf{I}}(t, \cdot)\right\|_{H^{1}(\mathbb{R})} \lesssim e^{-\varepsilon \gamma|\rho(t)|}+\varepsilon$. $\mathrm{u}$ 

let

$$
\mathcal{L} \bar{w}:=-\bar{w}_{y y}+c \bar{w}-p Q_{c}^{p-1}(y) \bar{w}, \quad \text { where } \quad Q_{c}(y):=c^{\frac{1}{p-1}} Q(\sqrt{c} y) .
$$

Here $\bar{w}=\bar{w}(y)$.

Lemma 3.3. Suppose that $A_{c}$ satisfies (3.4). Let $w$ given by (3.3). Then the following expansion holds:

$$
\begin{aligned}
\mathbf{I I}= & \left(c^{\prime}-\varepsilon f_{1}\right) \partial_{c} w-\left(\rho^{\prime}-c-\varepsilon f_{2}\right) w_{y}-(\mathcal{L} w)_{y} \\
& +\varepsilon^{2}\left[a_{0}^{\prime \prime} c A_{c}+f_{1} a_{0}^{\prime} \partial_{c} A_{c}\right]+\varepsilon^{2} F_{c}^{\mathbf{I I}}(t ; y),
\end{aligned}
$$

with

$$
\begin{aligned}
F_{c}^{\mathbf{I I}}(t ; \cdot)= & \varepsilon a_{0}^{\prime \prime}(\varepsilon \rho(t)) f_{2}(t) A_{c}+a_{0}^{\prime \prime}(\varepsilon \rho(t))\left(\rho^{\prime}(t)-c(t)-\varepsilon f_{2}(t)\right) A_{c} \\
& +\varepsilon^{-1} d(t) \partial_{t} A_{c}+O_{H^{1}(\mathbb{R})}\left(e^{-\varepsilon \gamma|\rho(t)|}\right),
\end{aligned}
$$

for some fixed $\gamma>0$.

REMARK 3.1. It turns out that the term $\varepsilon^{-1} d(t) \partial_{t} A_{c}$ will be a very problematic term to estimate; for a delicate treatment of this term see (3.39).

Proof. Let $D:=D_{c}(t, y), y=x-\rho(t)$, be a general, smooth function. We compute

$$
\mathbf{I I}(D):=D_{t}+\left(D_{x x}+p R^{p-1} D\right)_{x}+\varepsilon a_{0}^{\prime}(\varepsilon x) Q_{c_{0}} D
$$

We have

$$
\begin{aligned}
\mathbf{I I}(D)= & c^{\prime}(t) \partial_{c} D+D_{t}-\left(\rho^{\prime}(t)-c(t)\right) D_{y} \\
& +\left[D_{y y}-c(t) D+p Q_{c}^{p-1} D\right]_{x}+\varepsilon a_{0}^{\prime}(\varepsilon x) Q_{c_{0}} D \\
= & D_{t}-(\mathcal{L} D)_{y}+\left(c^{\prime}(t)-\varepsilon f_{1}(t)\right) \partial_{c} D \\
& -\left(\rho^{\prime}(t)-c(t)-\varepsilon f_{2}(t)\right) D_{y}+O\left(\varepsilon a_{0}^{\prime}(\varepsilon x) Q_{c_{0}} D\right) \\
& +\varepsilon f_{1}(t) \partial_{c} D-\varepsilon f_{2}(t) D_{y} .
\end{aligned}
$$

We apply this last identity to the function $w=\varepsilon d(t) A_{c}(t, y)$. We have

$$
\begin{aligned}
\mathbf{I I}(w)= & \varepsilon d^{\prime}(t) A_{c}+\varepsilon d(t) \partial_{t} A_{c}-\varepsilon d(t)\left(\mathcal{L} A_{c}\right)^{\prime}+\left(c^{\prime}(t)-\varepsilon f_{1}(t)\right) \varepsilon d(t) \partial_{c} A_{c} \\
& -\left(\rho^{\prime}(t)-c(t)-\varepsilon f_{2}(t)\right) \varepsilon d(t) A_{c}^{\prime}+\varepsilon^{2} d(t) f_{1}(t) \partial_{c} A_{c}+O_{H^{1}(\mathbb{R})}\left(\varepsilon^{2} e^{-\varepsilon \gamma|\rho(t)|}\right) \\
= & \varepsilon d(t)\left(c^{\prime}(t)-\varepsilon f_{1}(t)\right) \partial_{c} A_{c}-\varepsilon d(t)\left(\rho^{\prime}(t)-c(t)-\varepsilon f_{2}(t)\right) A_{c}^{\prime}-\varepsilon d(t)\left(\mathcal{L} A_{c}\right)^{\prime} \\
& +\varepsilon^{2}\left[\varepsilon^{-1} d^{\prime}(t) A_{c}+d(t) f_{1}(t) \partial_{c} A_{c}+\varepsilon^{-1} d(t) \partial_{t} A_{c}\right] \\
& +O_{H^{1}(\mathbb{R})}\left(\varepsilon^{2} e^{-\varepsilon \gamma|\rho(t)|}\right) .
\end{aligned}
$$

(Recall that $A_{c}^{\prime} \in \mathcal{S}$.) Now we use the fact that $d(t)=a_{0}^{\prime}(\varepsilon \rho(t))$ to compute $d^{\prime}(t)$. We have

$$
\begin{aligned}
d^{\prime}(t) & =\varepsilon a_{0}^{\prime \prime}(\varepsilon \rho(t)) \rho^{\prime}(t) \\
& =\varepsilon a_{0}^{\prime \prime}(\varepsilon \rho(t)) c(t)+\varepsilon^{2} a_{0}^{\prime \prime}(\varepsilon \rho(t)) f_{2}(t)+\varepsilon a_{0}^{\prime \prime}(\varepsilon \rho(t))\left(\rho^{\prime}(t)-c(t)-\varepsilon f_{2}(t)\right) .
\end{aligned}
$$

Replacing in (3.18) we conclude. 
Lemma 3.4. Suppose that $A_{c}$ satisfy 3.4). Then

$$
\mathbf{I I I}=O_{H^{1}(\mathbb{R})}\left(\varepsilon^{2} e^{-\varepsilon \gamma|\rho(t)|}\right) .
$$

Proof. First of all, define IIII $:=(R+w)^{p}-R^{p}-p R^{p-1} w$. Then,

$$
\tilde{\mathbf{I I}}= \begin{cases}\varepsilon^{2} d^{2}(t) A_{c}^{2} & \text { if } p=2 ; \\ \varepsilon^{2} d^{2}(t) A_{c}^{2}\left[3 Q_{c}+\varepsilon d(t) A_{c}\right] & \text { if } p=3 ; \\ \varepsilon^{2} d^{2}(t) A_{c}^{2}\left[6 Q_{c}^{2}+4 \varepsilon d(t) Q_{c} A_{c}+\varepsilon^{2} d^{2}(t) A_{c}^{2}\right] & \text { in the case } p=4 .\end{cases}
$$

Thus taking space derivative we obtain (3.19) (note that $\left(A_{c}^{p}\right)^{\prime} \in \mathcal{S}$ because $A_{c}$ satisfies (3.4) $)$.

Now we collect the estimates from Lemmas 3.2, 3.3 and 3.4. We obtain that, for all $t$ in a given interval,

$$
S[\tilde{u}]=\left(c^{\prime}(t)-\varepsilon f_{1}(t)\right) \partial_{c} \tilde{u}-\left(\rho^{\prime}(t)-c(t)-\varepsilon f_{2}(t)\right) \partial_{y} \tilde{u}+\tilde{S}[\tilde{u}],
$$

with

$(3.21) \tilde{S}[\tilde{u}]=\varepsilon\left[F_{1}(t, y)-d(t)\left(\mathcal{L} A_{c}\right)_{y}\right]$

$(3.22)+\varepsilon^{2}\left[a_{0}^{\prime \prime}(\varepsilon \rho(t)) c A_{c}+f_{1} a_{0}^{\prime}(\varepsilon \rho(t)) \partial_{c} A_{c}\right]+\varepsilon^{2} a_{0}^{\prime \prime}(\varepsilon \rho(t))\left(\rho^{\prime}(t)-c(t)-\varepsilon f_{2}(t)\right) A_{c}$

$(3.23)+\varepsilon d(t) \partial_{t} A_{c}+\varepsilon^{3} a_{0}^{\prime \prime}(\varepsilon \rho(t)) f_{2}(t) A_{c}+\varepsilon^{2} O_{H^{1}(\mathbb{R})}\left(e^{-\varepsilon \gamma|\rho(t)|}+\varepsilon\right)$.

In addition, $f_{1}(t), f_{2}(t)$ are given (for the moment) by (3.9)-(3.10), and

(3.24) $F_{1}:=F_{1}^{\mathbf{I}}=f_{1}(t) \Lambda Q_{c}-f_{2}(t) Q_{c}^{\prime}+a_{0}^{\prime}(\varepsilon \rho(t))\left[Q_{c}^{2}+\left(c_{0}-c\right) \Lambda Q_{c} Q_{c}+\left(\rho_{0}-\rho\right) Q_{c}^{\prime} Q_{c}\right]$,

(cf. (3.16)). Now we give an explicit value of $f_{1}(t)$, satisfying (3.9). It is not difficult to check that, for any $t$, there is a well-defined $f_{1}(t) \in \mathbb{R}$ such that

$$
\int_{\mathbb{R}} F_{1}(t, y) Q_{c}(y) d y=0 .
$$

More explicitly, using (3.5), we have

$$
\begin{aligned}
f_{1}(t) & =-a_{0}^{\prime}(\varepsilon \rho(t))\left(\int_{\mathbb{R}} \Lambda Q_{c} Q_{c}\right)^{-1} \int_{\mathbb{R}}\left[Q_{c}^{3}+\left(c_{0}-c\right) \Lambda Q_{c} Q_{c}^{2}\right] \\
& =f_{1}^{0}(t)-\frac{2(7-p)}{3(5-p)}\left(c_{0}-c\right) a_{0}^{\prime}(\varepsilon \rho(t)) c^{\frac{1}{p-1}} \frac{\int_{\mathbb{R}} Q^{3}}{\int_{\mathbb{R}} Q^{2}}
\end{aligned}
$$

with $f_{1}^{0}$ defined in (2.7). This and (3.2) proves (3.9).

The next step is the resolution of the linear differential equation involving the first order terms in $\varepsilon$. Indeed, from (3.21), we want to solve

$$
d(t)\left(\mathcal{L} A_{c}\right)_{y}(y)=F_{1}(t, y), \quad \text { for all } y \in \mathbb{R}, \text { and } t \text { fixed; }
$$

with $d(t)$ given by (3.3). Note that from (3.9)-(3.10) and (3.24) one has

$$
\begin{aligned}
F_{1}(t ; y) & :=a_{0}^{\prime}\left[-\lambda_{p} c^{\frac{p}{p-1}} \Lambda Q_{c}+Q_{c}^{2}+\left(c_{0}-c\right) \Lambda Q_{c} Q_{c}+\left(\rho_{0}-\rho\right) Q_{c}^{\prime} Q_{c}\right]-f_{2}(t) Q_{c}^{\prime}, \\
& =a_{0}^{\prime}\left[-\lambda_{p} c^{\frac{p}{p-1}} \Lambda Q_{c}+Q_{c}^{2}-\left(a_{0}^{\prime}\right)^{-1} f_{2} Q_{c}^{\prime}+\left(c_{0}-c\right) \Lambda Q_{c} Q_{c}+\left(\rho_{0}-\rho\right) Q_{c}^{\prime} Q_{c}\right] \\
(3.26) & =: d(t) \tilde{F}_{1}(t, y) .
\end{aligned}
$$


Therefore, we are reduced to solve the following simple problem,

$$
\left(\mathcal{L} A_{c}\right)_{y}(y)=\tilde{F}_{1}(t, y)
$$

with $\tilde{F}_{1}$ defined in (3.26), and from (3.25),

$$
\int_{\mathbb{R}} \tilde{F}_{1}(t, y) Q_{c}(y)=0 .
$$

Let us recall the following results (see e.g. [23]):

Lemma 3.5. The operator $\mathcal{L}$ defined $\left(\right.$ on $\left.L^{2}(\mathbb{R})\right)$ by (3.17) has domain $H^{2}(\mathbb{R})$, it is self-adjoint and satisfies the following properties:

1. The kernel of $\mathcal{L}$ is spanned by $Q_{c}^{\prime}$. Moreover, $\Lambda Q_{c}$ defined in (3.5) satisfies $\mathcal{L}\left(\Lambda Q_{c}\right)=-Q_{c}$. Finally, the continuous spectrum of $\mathcal{L}$ is given by $\sigma_{\text {cont }}(\mathcal{L})=$ $[c,+\infty)$.

2. For all $h=h(x)$ polynomially growing function such that $\int_{\mathbb{R}} h Q_{c}^{\prime}=0$, there exists a unique polynomially growing function $\hat{h}$ such that $\int_{\mathbb{R}} \hat{h} Q_{c}^{\prime}=0$ and $\mathcal{L} \hat{h}=h$. Moreover, if $h$ is even (resp. odd), then $\hat{h}$ is even (resp. odd).

3. For $h \in H^{2}(\mathbb{R}), \mathcal{L} h \in \mathcal{S}(\mathbb{R})$ implies $h \in \mathcal{S}(\mathbb{R})$.

Let $c>0$ and

$$
\varphi(x):=-\frac{Q^{\prime}(x)}{Q(x)}, \quad \varphi_{c}(x):=-\frac{Q_{c}^{\prime}}{Q_{c}}=\sqrt{c} \varphi(\sqrt{c} x) .
$$

Note that $\varphi$ is an odd function, with

$$
\lim _{x \rightarrow \pm \infty} \varphi(x)= \pm 1 ; \quad \varphi^{(k)} \in \mathcal{S}(\mathbb{R}), k \geq 1 .
$$

We recall the form of the solution $A_{c}$ that we are looking for. We seek for a bounded solution satisfying

$$
A_{c(t)}(t, y)=\beta_{c}(t)\left(\varphi_{c}(y)-\sqrt{c(t)}\right)+\hat{A}_{c}(t, y)+\mu_{c}(t) Q_{c}^{\prime}(y)+\delta_{c}(t) \Lambda Q_{c}(y)
$$

for some $\beta_{c}(t), \mu_{c}(t), \delta_{c}(t) \in \mathbb{R}, \varphi_{c}$ defined in (3.28), and $\hat{A}_{c}(t, \cdot) \in \mathcal{S}(\mathbb{R})$. The parameters $\mu_{c}$ and $\delta_{c}$ will be chosen in order to find the unique solution $A_{c}$ satisfying some orthogonality conditions.

Lemma 3.6. Suppose $(c(t), \rho(t))$ satisfying (2.5) and (3.2), and $f_{1}(t), f_{2}(t)$ given by (3.9)-(3.10). There exists a unique solution $A_{c}=A_{c(t)}(t, y)$ of

$$
\left(\mathcal{L} A_{c}\right)_{y}(t, y)=\tilde{F}_{1}(t, y),
$$

such that, for every $t$,

$$
\begin{aligned}
& A_{c}(t, y):=\beta_{c}(t)\left(\varphi_{c}(y)-\sqrt{c}\right)+\hat{A}_{c}(t, y)+\mu_{c}(t) Q_{c}^{\prime}(y)+\delta_{c}(t) \Lambda Q_{c}(y), \\
& \lim _{-\infty} A_{c}=-2 \sqrt{c} \beta_{c} ; \quad\left|A_{c}(y)\right| \leq K e^{-\gamma y}, \text { as } y \rightarrow+\infty
\end{aligned}
$$

with $\hat{A}_{c}(t) \in \mathcal{S}(\mathbb{R})$ for all $t$. In addition, we hav凹

$$
\beta_{c}(t):=\frac{1}{2 c^{3 / 2}(t)} \int_{\mathbb{R}} \tilde{F}_{1}(t, y) d y \neq 0, \quad\left|\beta_{c}(t)\right|+\left|\mu_{c}(t)\right|+\left|\delta_{c}(t)\right| \lesssim 1 .
$$

\footnotetext{
${ }^{4}$ Note that $\beta_{c}=0$ implies $A_{c} \in L^{2}(\mathbb{R})$.
} 
Finally, $A_{c}$ satisfies

$$
\int_{\mathbb{R}} A_{c}(t, y) Q_{c}(y) d y=\int_{\mathbb{R}} A_{c}(t, y) y Q_{c}(y) d y=0 .
$$

Proof. First of all, the existence of a solution $A_{c}(t, \cdot) \in L^{\infty}(\mathbb{R})$ of the form (3.32) for this equation was established in [28, provided

$$
\int_{\mathbb{R}} \tilde{F}_{1}(t, y) Q_{c}(y) d y=0,
$$

which is indeed the case (cf. (3.25)). Note that the inclusion of the term proportional to $f_{2}(t) Q_{c}^{\prime}$ in (3.24) induces the new term $\delta_{c} \Lambda Q_{c}$ in (3.32) (recall that from Lemma 3.5 $\left(\mathcal{L} \Lambda Q_{c}\right)^{\prime}=-Q_{c}^{\prime}$.) Furthermore, the limits in (3.33) are straightforward from (3.29).

Now, let us prove (3.34). Indeed, from (3.31), integrating over $\mathbb{R}$ and using (3.33), we get

$$
2 \beta_{c} c \sqrt{c}=c A_{c}(-\infty)=\mathcal{L} A_{c}(+\infty)-\mathcal{L} A_{c}(-\infty)=\int_{\mathbb{R}} \tilde{F}_{1},
$$

which gives the value of $\beta_{c}$, and the corresponding bound. Moreover,

$$
\begin{aligned}
\int_{\mathbb{R}} \tilde{F}_{1} & =c^{2 \theta} \int_{\mathbb{R}} Q^{2}-\lambda_{p} c^{2 \theta}\left(\frac{1}{p-1}-\frac{1}{2}\right) \int_{\mathbb{R}} Q+\left(c_{0}-c\right) \theta c^{2 \theta-1} \int_{\mathbb{R}} Q^{2} \\
& =c^{2 \theta}\left[\left(\int_{\mathbb{R}} Q^{2}\right)^{2}-\frac{2(3-p)}{(5-p)} \int_{\mathbb{R}} Q \int_{\mathbb{R}} Q^{3}\right]\left(\int_{\mathbb{R}} Q^{2}\right)^{-1}+O\left(\left|c_{0}-c\right|\right) \neq 0,
\end{aligned}
$$

for $p=3,4$. The case $p=2$ requires more care, but a simple computation gives a nonzero final value: note that from the identities $Q^{\prime \prime}=Q-Q^{2}$ and $Q^{\prime 2}=Q^{2}-\frac{2}{3} Q^{3}$, one has

$$
\int_{\mathbb{R}} Q=\int_{\mathbb{R}} Q^{2}, \quad \int_{\mathbb{R}} Q^{3}=\frac{6}{5} \int_{\mathbb{R}} Q^{2}=\frac{6}{5} \int_{\mathbb{R}} Q
$$

Therefore $\left(\int_{\mathbb{R}} Q^{2}\right)^{2}-\frac{2}{3} \int_{\mathbb{R}} Q \int_{\mathbb{R}} Q^{3}=\left(1-\frac{4}{5}\right)\left(\int_{\mathbb{R}} Q\right)^{2}>0$.

On the other hand, we choose the terms $\mu_{c}$ and $\delta_{c}$ in order to satisfy (3.35). The parameter $\mu_{c}(t)$ is chosen to satisfy the condition

$$
\int_{\mathbb{R}} y Q_{c} A_{c}=0
$$

and it does not give any problem. In order to deal with $\delta_{c}$, we need more information about $f_{2}(t)$. Since we do not explicitly know $A_{c}$, we need another method to compute an explicit expression for $f_{2}(t)$, satisfying (3.10) (and therefore, the corresponding bounds for $\delta_{c}(t)$ ). Indeed, multiplying (3.31) by $\int_{-\infty}^{y} \Lambda Q_{c} \in L^{\infty}(\mathbb{R})$ and integrating, one has

$$
\int_{\mathbb{R}}\left(\mathcal{L} A_{c}\right)_{y} \int_{-\infty}^{y} \Lambda Q_{c}=\int_{\mathbb{R}} \tilde{F}_{1} \int_{-\infty}^{y} \Lambda Q_{c}
$$

Integrating by parts, we get

$$
\left.\left(\mathcal{L} A_{c}\right) \int_{-\infty}^{y} \Lambda Q_{c}\right|_{-\infty} ^{+\infty}+\int_{\mathbb{R}}\left(\mathcal{L} A_{c}\right)_{y} \int_{-\infty}^{y} \Lambda Q_{c}=-\int_{\mathbb{R}} \Lambda Q_{c} \mathcal{L} A_{c}=\int_{\mathbb{R}} Q_{c} A_{c}=0 .
$$


Using (3.4), we have $\left.\left(\mathcal{L} A_{c}\right) \int_{-\infty}^{y} \Lambda Q_{c}\right|_{-\infty} ^{+\infty}=0$. Therefore, from (3.26),

$$
\begin{aligned}
& -f_{2} \int_{\mathbb{R}} Q_{c} \Lambda Q_{c}= \\
& \quad=a_{0}^{\prime} \int_{\mathbb{R}}\left[-\lambda_{p} c^{\frac{p}{p-1}} \Lambda Q_{c}+Q_{c}^{2}+\left(c_{0}-c\right) \Lambda Q_{c} Q_{c}+\left(\rho_{0}-\rho\right) Q_{c}^{\prime} Q_{c}\right] \int_{-\infty}^{y} \Lambda Q_{c} .
\end{aligned}
$$

A simple computation using the scaling of $Q_{c}, \Lambda Q_{c}$ and its derivatives, and integration by parts show that, for $\theta=\frac{1}{p-1}-\frac{1}{4}$,

$$
\begin{aligned}
-\theta f_{2} c^{2 \theta-1} \int_{\mathbb{R}} Q^{2}= & a_{0}^{\prime}\left[-\frac{1}{2} \lambda_{p} c^{\frac{p}{p-1}}\left(\int_{\mathbb{R}} \Lambda Q_{c}\right)^{2}+\int_{\mathbb{R}} Q_{c}^{2} \int_{-\infty}^{y} \Lambda Q_{c}\right. \\
& \left.+\left(c_{0}-c\right) \int_{\mathbb{R}} \Lambda Q_{c} Q_{c} \int_{-\infty}^{y} \Lambda Q_{c}-\frac{1}{2}\left(\rho_{0}-\rho\right) \int_{\mathbb{R}} Q_{c}^{2} \Lambda Q_{c}\right] \\
= & \frac{(3-p)}{2(p-1)} a_{0}^{\prime} c^{\frac{5-2 p}{p-1}}\left[-\frac{1}{4} \lambda_{p} \frac{(3-p)}{p-1}\left(\int_{\mathbb{R}} Q\right)^{2}+\int_{\mathbb{R}} Q^{2} \int_{-\infty}^{y} Q\right] \\
& +\left(c_{0}-c\right) a_{0}^{\prime} c^{\frac{3(2-p)}{p-1}} \int_{\mathbb{R}} Q \Lambda Q \int_{-\infty}^{y} \Lambda Q \\
& -\frac{(7-p)}{12(p-1)}\left(\rho_{0}-\rho\right) a_{0}^{\prime} c^{\frac{3(3-p)}{2(p-1)}} \int_{\mathbb{R}} Q^{3} .
\end{aligned}
$$

We finally obtain

$$
\begin{aligned}
& f_{2}(t)= \\
& \quad=\frac{2(p-3)}{(5-p)} a_{0}^{\prime}(\varepsilon \rho(t))[c(t)]^{\frac{2(5-2 p)}{7-3 p}}\left[-\frac{1}{4} \lambda_{p} \frac{(3-p)}{p-1}\left(\int_{\mathbb{R}} Q\right)^{2}+\int_{\mathbb{R}} Q^{2} \int_{-\infty}^{y} Q\right]\left(\int_{\mathbb{R}} Q^{2}\right)^{-1} \\
& \quad-\frac{5-p}{4(p-1)}\left(c_{0}(t)-c(t)\right) a_{0}^{\prime}(\varepsilon \rho(t))[c(t)]^{\frac{6(2-p)}{7-3 p}}\left(\int_{\mathbb{R}} Q \Lambda Q \int_{-\infty}^{y} \Lambda Q\right)\left(\int_{\mathbb{R}} Q^{2}\right)^{-1} \\
& \quad+\frac{(7-p)}{3(5-p)}\left(\rho_{0}(t)-\rho(t)\right) a_{0}^{\prime}(\varepsilon \rho(t))[c(t)]^{\frac{3(3-p)}{7-3 p}}\left(\int_{\mathbb{R}} Q^{3}\right)\left(\int_{\mathbb{R}} Q^{2}\right)^{-1} \\
& =: \mu_{p} a_{0}^{\prime}(\varepsilon \rho(t))[c(t)]^{\frac{2(5-2 p)}{7-3 p}}+O\left(a_{0}^{\prime}(\varepsilon \rho(t))\left(\left|c_{0}(t)-c(t)\right|+\left|\rho_{0}(t)-\rho(t)\right|\right)\right),
\end{aligned}
$$

as desired (cf. (3.10) and (2.8), and note that $\mu_{3}=0$ ). Note that from (3.10) we have that $\delta_{c}(t)$ satisfies the required estimates.

口

Having solved the linear problem, from (3.20) and (3.21) we have

$$
S[\tilde{u}](t, x)=\left(c^{\prime}(t)-\varepsilon f_{1}(t)\right) \partial_{c} \tilde{u}-\left(\rho^{\prime}(t)-c(t)-\varepsilon f_{2}(t)\right) \partial_{y} \tilde{u}+\tilde{S}[\tilde{u}](t, x),
$$

where $\tilde{S}[\tilde{u}]$ given in $(3.22)-(3.23)$ will be of second order in $\varepsilon$, as we show in the following lines.

Let us describe the dependence on $c$ and $t$ of the solution $A_{c}$. From (3.26) (see also Lemma 4.5 in [28]), one has

$$
\tilde{F}_{1}(t, y)=c^{\frac{2}{p-1}} \tilde{F}_{1}^{1}(\sqrt{c} y)+c^{\frac{32 p-13-11 p^{2}}{2(p-1)(7-3 p)}} \tilde{F}_{1}^{2}(\sqrt{c} y)+O_{H^{1}(\mathbb{R})}\left(\left|c-c_{0}\right|+\left|\rho-\rho_{0}\right|\right),
$$

\footnotetext{
${ }^{5}$ Note that the exponents $\frac{6(2-p)}{7-3 p}$ and $\frac{3(3-p)}{7-3 p}$ are both nonnegative for $p=2,3$ and 4 .
} 
where

$$
\tilde{F}_{1}^{1}(y):=-\lambda_{p} \Lambda Q(y)+Q^{2}(y), \quad \tilde{F}_{1}^{2}(y):=Q^{\prime} .
$$

Therefore, Claim 3 in 28] allows to conclude that $A_{c}$ satisfies the following decomposition:

$$
A_{c}(t, y)=c^{\frac{7-3 p}{2(p-1)}} \tilde{A}_{1}^{1}(t, \sqrt{c} y)+c^{\frac{4+p-p^{2}}{(p-1)(7-3 p)}} \tilde{A}_{1}^{2}(t, \sqrt{c} y)+O_{L^{\infty}(\mathbb{R})}\left(\left|c-c_{0}\right|+\left|\rho-\rho_{0}\right|\right),
$$

with $\tilde{A}_{1}^{1}$ bounded solution of $\left(\mathcal{L} \tilde{A}_{1}^{1}\right)^{\prime}=\tilde{F}_{1}^{1}$, and $\tilde{A}_{1}^{2}(\sqrt{c} y) \in \mathcal{S}(\mathbb{R})$. Moreover, one has $\left(\tilde{A}_{1}^{1}\right)^{\prime} \in \mathcal{S}(\mathbb{R})$. Using this decomposition we have that, avoiding the terms proportional to $\left|c-c_{0}\right|, \partial_{c} A_{c}$ has the same behavior as $A_{c}$ : it is bounded, it is not $L^{2}$-integrable, and satisfies $\lim _{+\infty} \partial_{c} A_{c}=0, \lim _{-\infty} \partial_{c} A_{c} \neq 0$. The same result holds for $\partial_{c}^{2} A_{c}$.

We consider now the term $\partial_{t} A_{c}$, avoiding the terms with usual derivatives with respect to $c$ and $\rho$. In fact, $\partial_{t} A_{c}(t, y)$ involves derivatives with respect to $t$ of $\left(c_{0}-c\right)$ and $\left(\rho_{0}-\rho\right)$. More specifically, from the explicit composition of $F_{1}$ in (3.26) the solution $A_{c}$ can be decomposed as follows

$$
\begin{aligned}
A_{c}(t, y)= & A_{c, s}(t, y)+a_{0}^{\prime}(\varepsilon \rho(t))\left(\rho_{0}(t)-\rho(t)\right) D_{c}(t, y) \\
& +a_{0}^{\prime}(\varepsilon \rho(t))\left(c_{0}(t)-c(t)\right) E_{c}(t, y),
\end{aligned}
$$

where $A_{c, s}$ is the solution of

$$
\left(\mathcal{L} A_{c, s}\right)_{y}=a_{0}^{\prime}\left[-\lambda_{p} c^{\frac{p}{p-1}} \Lambda Q_{c}+Q_{c}^{2}\right],
$$

$D_{c}$ solves

$$
\mathcal{L} D_{c}=\frac{1}{2} Q_{c}^{2}-\left(a_{0}^{\prime}\right)^{-1} f_{2} Q_{c}
$$

and $E_{c}$ is the solution of

$$
\left(\mathcal{L} E_{c}\right)_{y}=\Lambda Q_{c} Q_{c} .
$$

It is clear that $D_{c}(t, \cdot) \in \mathcal{S}(\mathbb{R})$ and $E_{c}(t, \cdot) \in L^{\infty}(\mathbb{R})$. Since the term $A_{c, s}(t, y)$ only contains derivatives in time already computed in (3.20), we get

$$
\partial_{t} A_{c}=a_{0}^{\prime}(\varepsilon \rho(t))\left(\rho_{0}^{\prime}(t)-\rho^{\prime}(t)\right) D_{c}(t, y)+a_{0}^{\prime}(\varepsilon \rho(t))\left(c_{0}^{\prime}(t)-c^{\prime}(t)\right) E_{c}(t, y) .
$$

Note that

$$
\begin{aligned}
c_{0}^{\prime}-c^{\prime} & =\varepsilon\left(f_{1}^{0}\left(c_{0}, \rho_{0}\right)-f_{1}(c, \rho)\right)+O\left(\left|c^{\prime}-\varepsilon f_{1}\right|\right) \\
& =O\left(\varepsilon e^{-\gamma \varepsilon\left|\rho_{0}\right|}+\varepsilon e^{-\gamma \varepsilon|\rho|}+\left|c^{\prime}-\varepsilon f_{1}\right|\right) .
\end{aligned}
$$

Note that the second term can be added to the dynamical system 3.20 without perturbing the dynamics. The worst case is with no doubt the first one. We have

$$
\left|\rho_{0}^{\prime}-\rho^{\prime}\right| \lesssim\left|c_{0}-c\right|+\left|\rho^{\prime}-c-\varepsilon f_{2}\right|+O(\varepsilon) .
$$

The term $\left|\rho^{\prime}-c-\varepsilon f_{2}\right|$ can be added to the dynamical system (3.20) as in the previous case. In concluding, without considering the terms proportional to $\left|\rho^{\prime}-c-\varepsilon f_{2}\right|$ and $\left|c^{\prime}-\varepsilon f_{1}\right|$,

$$
\begin{aligned}
\tilde{S}[\tilde{u}] & =(3.22)+(3.23) \\
& =O\left(\varepsilon^{2} e^{-\gamma \varepsilon\left|\rho_{0}\right|}+\varepsilon^{2} e^{-\gamma \varepsilon|\rho|}\right)+\varepsilon a_{0}^{\prime}(\varepsilon \rho(t))\left(c_{0}(t)-c(t)\right) D_{c}(t, y),
\end{aligned}
$$


with exponential decay as $y \rightarrow+\infty$, and $D(t, \cdot) \in \mathcal{S}(\mathbb{R})$. These estimates will be useful when computing (4.34).

Let us conclude the proof of Proposition 3.1. Using the decay on the right of $A_{c}$ (see (3.33)), estimate (3.11) is direct. In addition, from Lemma 3.6 we have (3.4), and $f_{1}(t)$ and $f_{2}(t)$ are well determined by (3.9)-(3.10). Finally, from (3.22)-(3.23) one has (3.12). These facts prove Proposition 3.1.

प

The next results are similar to those proved in [28, 29, but for the sake of completeness, we include them. Recall that $\tilde{u}$ does not belong to $L^{2}(\mathbb{R})$. In order to solve this problem, consider a cut-off function $\eta \in C^{\infty}(\mathbb{R})$ satisfying the following properties:

$$
\left\{\begin{array}{l}
0 \leq \eta(s) \leq 1, \quad 0 \leq \eta^{\prime}(s) \leq 1, \quad \text { for any } s \in \mathbb{R} \\
\eta(s) \equiv 0 \text { for } s \leq-1, \quad \eta(s) \equiv 1 \text { for } s \geq 1
\end{array}\right.
$$

and define

$$
\eta_{\varepsilon}(y):=\eta(\varepsilon y+2)
$$

and for $w=w(t, y)$ the first order correction constructed in Lemma 3.6, redefine

$$
\tilde{u}(t, x):=\eta_{\varepsilon}(y) \tilde{u}(t, x)=\eta_{\varepsilon}(y)(R(t, x)+w(t, x)),
$$

and similarly for $R(t)$ and $w(t)$. Note that, by definition,

$$
\tilde{u}(t, x)=0 \quad \text { for all } y \leq-\frac{3}{\varepsilon} .
$$

The following Proposition deals with the error associated to this cut-off function, and the new approximate solution $\tilde{u}$.

Proposition 3.7. There exist constants $\varepsilon_{0}, \gamma>0$ such that for all $0<\varepsilon<\varepsilon_{0}$ the following holds.

1. Consider the localized function $\tilde{u}(t)=R(t)+w(t)$ defined in 3.41)-3.42, for $t$ in a given interval. Then we have

(a) $L^{2}$-solution. $w(t, \cdot) \in H^{1}(\mathbb{R})$, with

$$
\|w(t, \cdot)\|_{H^{1}(\mathbb{R})} \lesssim \sqrt{\varepsilon} e^{-\gamma \varepsilon|\rho(t)|} .
$$

(b) Almost orthogonality.

$$
\left|\int_{\mathbb{R}} w(t, x) Q_{c}(y) d x\right|+\left|\int_{\mathbb{R}} y w(t, x) Q_{c}(y) d x\right| \lesssim \varepsilon^{10} .
$$

2. Almost solution. The error associated to the new function $\tilde{u}(t)$ satisfies

$$
\begin{aligned}
S[\tilde{u}]= & \left(c^{\prime}(t)-\varepsilon f_{1}(t)\right)\left(\partial_{c} \tilde{u}+O_{H^{1}}\left(\varepsilon a_{0}^{\prime}(\varepsilon \rho)\left|c_{0}-c\right|\right)\right) \\
& -\left(\rho^{\prime}(t)-c(t)-\varepsilon f_{2}(t)\right)\left(\tilde{u}_{y}+O_{H^{1}}\left(\varepsilon a_{0}^{\prime}(\varepsilon \rho)\left(\left|c_{0}-c\right|+\left|\rho_{0}-\rho\right|\right)\right)\right) \\
& +\tilde{S}[\tilde{u}](t),
\end{aligned}
$$

with

$$
\left\|\tilde{S}[\tilde{u}](t)-\varepsilon a_{0}^{\prime}(\varepsilon \rho(t))\left(c_{0}(t)-c(t)\right) D_{c}(t)\right\|_{H^{1}(\mathbb{R})} \lesssim \varepsilon^{3 / 2} e^{-\gamma \varepsilon|\rho(t)|} .
$$

Finally, one has (3.12). 
Proof. The proof of (3.44) follows from a direct computation. Indeed,

$$
\left\|w(t) \eta_{\varepsilon}\right\|_{H^{1}(\mathbb{R})} \lesssim\|w(t)\|_{H^{1}\left(y \geq-\frac{3}{\varepsilon}\right)},
$$

but from (3.4),

$$
\left\|\varepsilon d(t) A_{c}(y)\right\|_{H^{1}\left(y \geq-\frac{3}{\varepsilon}\right)} \lesssim \sqrt{\varepsilon} e^{-\varepsilon \gamma|\rho(t)|} .
$$

Let us now consider (3.45). Here we have, using (3.4),

$$
\int_{\mathbb{R}} w(t, x) \eta_{\varepsilon}(y) Q_{c}(y)=\int_{\mathbb{R}} w(t, x)(\eta(\varepsilon y+2)-1) Q_{c}(y) .
$$

Note that $\eta(\varepsilon y+2)-1 \equiv 0$ for $y \geq-\frac{1}{\varepsilon}$. Using the exponential decay of $Q_{c}(y)$, we have

$$
\begin{aligned}
\left|\int_{\mathbb{R}} w(t, x) \eta_{\varepsilon}(y) Q_{c}(y)\right| & \lesssim \int_{y \leq-\frac{2}{\varepsilon}} \varepsilon|y| e^{\sqrt{c} y}+\int_{y \in\left(-\frac{2}{\varepsilon},-\frac{1}{\varepsilon}\right)} \varepsilon|y| e^{-\frac{1}{2}(\varepsilon y+2)} e^{\sqrt{c} y} \\
& \lesssim e^{-\gamma / \varepsilon} \lesssim \varepsilon^{10} .
\end{aligned}
$$

The proof for $y A_{c}$ is very similar. We skip the details. For the proof of (3.46), we proceed as follows. First of all, a simple computation shows that

$$
S\left[\eta_{\varepsilon} \tilde{u}\right]=\eta_{\varepsilon} S[\tilde{u}]+\left(\eta_{\varepsilon}\right)_{t} \tilde{u}+3 \varepsilon \eta_{\varepsilon}^{\prime} \tilde{u}_{x x}+3 \varepsilon^{2} \eta_{\varepsilon}^{(2)} \tilde{u}_{x}+\varepsilon^{3} \eta_{\varepsilon}^{(3)} \tilde{u}+\varepsilon\left(\eta_{\varepsilon}^{p}\right)^{\prime} \tilde{u}^{p} .
$$

Since $\operatorname{supp} \eta_{\varepsilon}^{(k)} \subseteq\left[-\frac{3}{\varepsilon},-\frac{1}{\varepsilon}\right]$ for $k=1,2$ and 3 , we have

$$
3 \varepsilon \eta_{\varepsilon}^{\prime} \tilde{u}_{x x}+3 \varepsilon^{2} \eta_{\varepsilon}^{(2)} \tilde{u}_{x}+\varepsilon^{3} \eta_{\varepsilon}^{(3)} \tilde{u}+\varepsilon\left(\eta_{\varepsilon}^{p}\right)^{\prime} \tilde{u}^{p}=O_{H^{1}(\mathbb{R})}\left(\varepsilon^{3 / 2} e^{-\varepsilon \gamma|\rho(t)|}\right)+O_{H^{1}(\mathbb{R})}\left(\varepsilon^{10}\right) .
$$

Similarly, from the definition of $\rho^{\prime}(t)$ and (2.5)

$$
\left(\eta_{\varepsilon}\right)_{t} \tilde{u}=-\rho^{\prime}(t) \varepsilon \eta_{\varepsilon}^{\prime} \tilde{u}=O_{H^{1}(\mathbb{R})}\left(\varepsilon^{3 / 2} e^{-\varepsilon \gamma|\rho(t)|}\right)+O_{H^{1}(\mathbb{R})}\left(\varepsilon^{10}\right) .
$$

Collecting the terms above, we have

$$
S\left[\eta_{\varepsilon} \tilde{u}\right]=\eta_{\varepsilon} S[\tilde{u}]+O_{H^{1}(\mathbb{R})}\left(\varepsilon^{3 / 2} e^{-\varepsilon \gamma|\rho(t)|}\right)+O_{H^{1}(\mathbb{R})}\left(\varepsilon^{10}\right) .
$$

Finally, from the decomposition (3.8), one has $S[\tilde{u}]=$ dynamical system $+\tilde{S}[\tilde{u}]$, and from (3.46), (3.3) and (3.4),

$$
\left\|\eta_{\varepsilon} \tilde{S}[\tilde{u}]\right\|_{H^{1}(\mathbb{R})} \lesssim \varepsilon^{3 / 2} e^{-\varepsilon \gamma|\rho(t)|}+\varepsilon\left|c(t)-c_{0}(t)\right| e^{-\varepsilon \gamma|\rho(t)|}+\varepsilon^{3} .
$$

Note that the proof of (3.12) does not vary at all. Finally, one has

$$
\begin{aligned}
\eta_{\varepsilon}\left[\left(c^{\prime}-\varepsilon f_{1}\right) \partial_{c} \tilde{u}+\left(\rho^{\prime}-c-\varepsilon f_{2}\right) \partial_{\rho} \tilde{u}\right]= & \left(c^{\prime}-\varepsilon f_{1}\right) \partial_{c}\left(\eta_{\varepsilon} \tilde{u}\right)-\left(\rho^{\prime}-c-\varepsilon f_{2}\right) \partial_{y}\left(\eta_{\varepsilon} \tilde{u}\right) \\
& +\varepsilon\left(\rho^{\prime}-c-\varepsilon f_{2}\right) \eta_{\varepsilon}^{\prime} \tilde{u} .
\end{aligned}
$$

Since $\varepsilon \eta_{\varepsilon}^{\prime} \tilde{u}=O_{H^{1}(\mathbb{R})}\left(\varepsilon^{3 / 2} e^{-\varepsilon \gamma|\rho(t)|}\right)$, from this last estimate, we get the final conclusion. $\square$ Finally, we recall that

$$
\left\|\tilde{S}[\tilde{u}](t)-\varepsilon a_{0}^{\prime}(\varepsilon \rho(t))\left(c_{0}(t)-c(t)\right) D_{c}(t, y)\right\|_{H^{1}(\mathbb{R})} \lesssim \varepsilon^{3 / 2} e^{-\varepsilon \gamma|\rho(t)|} .
$$

with $\left|D_{c}(t, y)\right|+\left|\partial_{x} D_{c}(t, y)\right| \lesssim e^{-\gamma_{0}|y|}$, for some fixed constant $\gamma_{0}>0$. 
4. Lyapunov stability. In this section we prove the following

Proposition 4.1. The following holds for any $0<\varepsilon<\varepsilon_{0}$. There exist $K_{0}>0$ independent of $\varepsilon$ and unique $C^{1}$ functions $c, \rho:[0, T] \rightarrow \mathbb{R}$ such that, for all $t \in[0, T]$,

$$
\begin{aligned}
|| u(t)-\tilde{u}(t, c(t), \rho(t)) \|_{H^{1}(\mathbb{R})} & \leq K_{0} \sqrt{\varepsilon}, \\
\left|c(t)-c_{0}(t)\right|+\left|\rho(t)-\rho_{0}(t)\right| & \lesssim K_{0} \sqrt{\varepsilon},
\end{aligned}
$$

and

$$
\left|\rho^{\prime}(t)-c(t)-\varepsilon f_{2}(t)\right|+\frac{1}{\sqrt{\varepsilon}}\left|c^{\prime}(t)-\varepsilon f_{1}(t)\right| \lesssim K_{0} \sqrt{\varepsilon} .
$$

Finally, one has

$$
|c(0)-1|+\left|\rho(0)+\varepsilon^{1+\delta_{0}}\right| \lesssim \sqrt{\varepsilon},
$$

with constants independent of $K_{0}$.

A direct conclusion of the previous result is the following

Corollary 4.2. Let $T_{0}>0$ be the maximal time of existence of $u(t)$. Then

$$
T_{0} \geq \varepsilon^{-1-\delta_{0}} .
$$

REMARK 4.1 (Notation). For the sake of brevity, in the forthcoming computations, we will denote

$$
c_{1}^{\prime}:=c^{\prime}-\varepsilon f_{1}, \quad \text { and } \quad \rho_{1}^{\prime}:=\rho^{\prime}-c-\varepsilon f_{2} .
$$

Proof. [Proof of Proposition 4.1] Let $K^{*}>1$ be a constant to be fixed later. Since $u(0)=Q(x)$, by the local continuity in $H^{1}(\mathbb{R})$ of the $g K d V$ flow, there exists a time $T^{*}>0$ such that, for all $t \in\left[0, T^{*}\right]$, we can find continuous functions $\lambda(t), r(t) \in \mathbb{R}$, such that

$$
\begin{gathered}
\|u(t)-\tilde{u}(\cdot, \lambda(t), r(t))\|_{H^{1}(\mathbb{R})} \leq K^{*} \sqrt{\varepsilon}, \\
\left|\lambda(t)-c_{0}(t)\right|+\left|r(t)-\rho_{0}(t)\right| \lesssim K^{*} \varepsilon^{1 / 2\left(1-\delta_{0}\right)},
\end{gathered}
$$

and

$$
|\lambda(0)-1|+\left|r(0)+\varepsilon^{1+\delta_{0}}\right| \lesssim \sqrt{\varepsilon},
$$

with a constant independent of $K^{*}$ large. Without loss of generality, we can assume $T^{*}=T^{*}\left(K^{*}\right)$ as the least upper bound of times such that the properties above are satisfied. The objective is to prove that we can take $T^{*} \geq T$ for $K^{*}$ large enough, by proving a bootstrap estimate for suitable well chosen parameters $\lambda(t), r(t)$. Our first step is to choose such parameters.

Lemma 4.3. Assume $0<\varepsilon<\varepsilon_{0}\left(K^{*}\right)$ small enough. There exist unique $C^{1}$ functions $c(t), \rho(t)$ such that, for all $t \in\left[0, T^{*}\right]$,

$$
z(t)=u(t)-\tilde{u}(t, c(t), \rho(t)) \text { satisfies } \int_{\mathbb{R}} z(t, x) y Q_{c}(y) d x=\int_{\mathbb{R}} z(t, x) Q_{c}(y) d x=0 .
$$


Moreover, we have, for all $t \in\left[0, T^{*}\right]$,

$$
\begin{gathered}
\|z(0)\|_{H^{1}(\mathbb{R})}+|c(0)-1|+\left|\rho(0)+\varepsilon^{1+\delta_{0}}\right| \lesssim \sqrt{\varepsilon} \\
\|z(t)\|_{H^{1}(\mathbb{R})} \lesssim K^{*} \sqrt{\varepsilon}, \quad\left|c(t)-c_{0}(t)\right|+\left|\rho(t)-\rho_{0}(t)\right| \lesssim K^{*} \sqrt{\varepsilon} .
\end{gathered}
$$

In addition, $z(t)$ satisfies the following equation

$$
z_{t}+\left\{z_{x x}+(\tilde{u}+z)^{m}-\tilde{u}^{m}\right\}_{x}+\varepsilon a_{0}^{\prime}(\varepsilon x) Q_{c_{0}} z+\tilde{S}[\tilde{u}]+c_{1}^{\prime}(t) \partial_{c} \tilde{u}-\rho_{1}^{\prime}(t) \partial_{y} \tilde{u}=0 .
$$

Finally, there exists $\gamma>0$ independent of $K^{*}$ such that for every $t \in\left[0, T^{*}\right]$,

$$
\left|\rho_{1}^{\prime}(t)\right| \lesssim\left[\int_{\mathbb{R}} z^{2} e^{-\gamma \sqrt{c}|y|}\right]^{1 / 2}+\int_{\mathbb{R}} e^{-\gamma \sqrt{c}|y|} z^{2}(t)+\left|\int_{\mathbb{R}} y Q_{c} \tilde{S}[\tilde{u}]\right|,
$$

and

$$
\left|c_{1}^{\prime}(t)\right| \lesssim \int_{\mathbb{R}} e^{-\gamma \sqrt{c}|y|} z^{2}(t)+\varepsilon e^{-\gamma \varepsilon|\rho(t)|}\left[\int_{\mathbb{R}} e^{-\gamma \sqrt{c}|y|} z^{2}(t)\right]^{1 / 2}+\left|\int_{\mathbb{R}} Q_{c} \tilde{S}[\tilde{u}]\right|
$$

Proof. The proof of (4.6)-(4.7) is a standard consequence of the Implicit Function Theorem, applied for each time $t \in\left[0, T^{*}\right]$. Indeed, for fixed $t \in[0, T]$, let us define the map

$$
(v, c, \rho) \in H^{1}(\mathbb{R}) \times \mathbb{R}_{+} \times \mathbb{R} \longmapsto J(v, c, \rho) \in \mathbb{R}^{2},
$$

where $J=\left(J_{1}, J_{2}\right)$ and

$$
\begin{aligned}
& J_{1}(v, c, \rho):=\int_{\mathbb{R}}(v-\tilde{u}(t, c, \rho))(x-\rho) Q_{c}(x-\rho) d x, \\
& J_{2}(v, c, \rho):=\int_{\mathbb{R}}(v-\tilde{u}(t, c, \rho)) Q_{c}(x-\rho) d x .
\end{aligned}
$$

It is clear that

$$
J_{1}\left(\tilde{u}\left(t, c_{0}(t), \rho_{0}(t)\right), c_{0}(t), \rho_{0}(t)\right)=J_{2}\left(\tilde{u}\left(t, c_{0}(t), \rho_{0}(t)\right), c_{0}(t), \rho_{0}(t)\right) \equiv 0 .
$$

Moreover, the respective Jacobian determinant of $J$ with respect to the variables $(c, \rho)$ is nonzero everywhere. Therefore, from the Implicit Function Theorem, there exists a small $\eta_{0}(t)>0$ (which can be chosen continuous on $t$ ) such that, for all $v \in H^{1}(\mathbb{R})$ satisfying $\left\|v-\tilde{u}\left(t, c_{0}(t), \rho_{0}(t)\right)\right\|_{H^{1}(\mathbb{R})}<\eta_{0}$, there is a smooth pair of parameters $(c(v), \rho(v)) \in \mathbb{R}^{2}$, satisfying $J(v, c(v), \rho(v)) \equiv 0$. Since the interval $\left[0, T^{*}\right]$ is compact, we can ensure $\eta_{0}>0$ independent of $t$.

Note that from (4.4)-(4.5), the function $u(t)$ satisfies, for each $t \in[0, T]$,

$$
\left\|u(t)-\tilde{u}\left(t, c_{0}(t), \rho_{0}(t)\right)\right\|_{H^{1}(\mathbb{R})} \lesssim K_{0} \sqrt{\varepsilon} \ll \eta_{0},
$$

provided $\varepsilon_{0}=\varepsilon_{0}\left(\eta_{0}\right)$ is chosen even smaller. Therefore there exists a smooth pair of parameters $(c(u(t)), \rho(u(t)))=:(c(t), \rho(t)) \in \mathbb{R}^{2}$, such that $J(u(t), c(t), \rho(t)) \equiv 0$. This proves 4.6).

The proof of (4.7) is direct from the initial condition $u_{0}(x)=Q(x)$, and (2.5). Finally, (4.8) follows from (4.12) and the fact that $J(u(t), c(t), \rho(t)) \equiv 0$. 
On the other hand, (4.9) is a direct computation. For the proof of (4.10) and (4.11), see e.g. [29]. $\square$ From (3.12) and (4.7), a crude estimate of the parameter $\left|c_{1}^{\prime}(t)\right|$ gives $\left|c_{1}^{\prime}(t)\right| \lesssim \varepsilon$, so that

$$
\int_{0}^{T^{*}}\left|c_{1}^{\prime}(t)\right| d t \lesssim \varepsilon^{-\delta_{0}}
$$

which is not a good estimate. In the following lines, we prove a sharp Virial estimate [22, 29] which allows to improve the preceding bound.

First of all, we define some auxiliary functions. Let $\phi \in C^{\infty}(\mathbb{R})$ be an even function satisfying the following properties

$$
\left\{\begin{array}{l}
\phi^{\prime} \leq 0 \text { on }[0,+\infty) ; \quad \phi(x)=1 \text { on }[0,1] \\
\phi(x)=e^{-x} \text { on }[2,+\infty) \text { and } e^{-x} \leq \phi(x) \leq 3 e^{-x} \text { on }[0,+\infty) .
\end{array}\right.
$$

Now, set $\psi(x):=\int_{0}^{x} \phi$. It is clear that $\psi$ an odd function. Moreover, for $|x| \geq 2$,

$$
\psi(+\infty)-\psi(|x|)=e^{-|x|}
$$

Finally, for $A>0$, denote

$$
\psi_{A}(x):=A \psi\left(\frac{x}{A}\right) ; \quad e^{-|x| / A} \leq \psi_{A}^{\prime}(x) \leq 3 e^{-|x| / A} .
$$

We claim the following

Lemma 4.4. There exist $K, A_{0}, \delta_{0}>0$ such that for all $t \in\left[0, T^{*}\right]$,

$$
\partial_{t} \int_{\mathbb{R}} z^{2}(t, x) \psi_{A_{0}}(y) \leq-\delta_{0} \int_{\mathbb{R}}\left(z_{x}^{2}+z^{2}\right)(t, x) e^{-\frac{1}{A_{0}}|y|}+K A_{0}\left(K^{*}\right)^{p+1} \varepsilon^{5 / 2} .
$$

Proof. Let $t \in\left[0, T^{*}\right]$. Replacing the value of $z_{t}$ given by (4.9), we have

$$
\begin{aligned}
\partial_{t} \int_{\mathbb{R}} z^{2} \psi_{A_{0}}(y)= & 2 \int_{\mathbb{R}} z z_{t} \psi_{A_{0}}(y)-\rho^{\prime}(t) \int_{\mathbb{R}} z^{2} \psi_{A_{0}}^{\prime}(y) \\
= & -2 \varepsilon \int_{\mathbb{R}} a_{0}^{\prime}(\varepsilon x) Q_{c_{0}} z^{2} \psi_{A_{0}}(y)+2 \int_{\mathbb{R}}\left(z \psi_{A_{0}}(y)\right)_{x}\left(z_{x x}+p R^{p-1} z\right) \\
& -\left(c+\varepsilon f_{2}\right)(t) \int_{\mathbb{R}} z^{2} \psi_{A_{0}}^{\prime}-2 \rho_{1}^{\prime}(t) \int_{\mathbb{R}} z \partial_{\rho} \tilde{u} \psi_{A_{0}} \\
& +2 \int_{\mathbb{R}}\left(z \psi_{A_{0}}(y)\right)_{x}\left[(\tilde{u}+z)^{p}-\tilde{u}^{p}-p \tilde{u}^{p-1} z\right] \\
& -2 c_{1}^{\prime}(t) \int_{\mathbb{R}} z \partial_{c} \tilde{u} \psi_{A_{0}}-\rho_{1}^{\prime}(t) \int_{\mathbb{R}} z^{2} \psi_{A_{0}}^{\prime} \\
& +2 p \int_{\mathbb{R}} z\left(z \psi_{A_{0}}(y)\right)_{x}\left(\tilde{u}^{p-1}-R^{p-1}\right)-2 \int_{\mathbb{R}} z \psi_{A_{0}} \tilde{S}[\tilde{u}] .
\end{aligned}
$$

The first term in (4.17) can be estimated as follows:

$$
\left|\varepsilon \int_{\mathbb{R}} a_{0}^{\prime}(\varepsilon x) Q_{c_{0}} z^{2} \psi_{A_{0}}(y)\right| \lesssim \varepsilon A_{0} \int_{\mathbb{R}} z^{2}(t) e^{-\frac{1}{A_{0}}|y|} .
$$


On the other hand, note that

$$
\begin{aligned}
|[4.19)| \lesssim & \left|\int_{\mathbb{R}} z_{x} \psi_{A_{0}}(y)\left[(\tilde{u}+z)^{p}-\tilde{u}^{p}-p \tilde{u}^{p-1} z\right]\right| \\
& \quad+\left|\int_{\mathbb{R}} \psi_{A_{0}}^{\prime}(y) z\left[(\tilde{u}+z)^{p}-\tilde{u}^{p}-p \tilde{u}^{p-1} z\right]\right| \\
\lesssim & A_{0} K^{*} \varepsilon^{1 / 2} \int_{\mathbb{R}} z^{2}(t) e^{-\gamma \sqrt{c}|y|}+K^{*} \varepsilon^{1 / 2} \int_{\mathbb{R}} z^{2}(t) e^{-\frac{1}{A_{0}}|y|}+\left|\int_{\mathbb{R}} z^{p+1} \psi_{A_{0}}^{\prime}(y)\right| \\
\lesssim & K^{*} A_{0} \varepsilon^{1 / 2} \int_{\mathbb{R}} z^{2}(t) e^{-\frac{1}{A_{0}}|y|}+A_{0} \varepsilon\|z(t)\|_{H^{1}(\mathbb{R})}^{p+1} \\
\lesssim & K^{*} A_{0} \varepsilon^{1 / 2} \int_{\mathbb{R}} z^{2}(t) e^{-\frac{1}{A_{0}}|y|}+\left(K^{*}\right)^{p+1} A_{0} \varepsilon^{(p+3) / 2} .
\end{aligned}
$$

for $A_{0}$ large, but independent of $\varepsilon$. Now, by using (4.10) and (4.11) it is easy to check that for $A_{0}$ large enough, and some constants $\delta_{0}, \varepsilon_{0}$ small, one has

$$
\begin{aligned}
|\underline{(4.20)}| & \lesssim\left|c_{1}^{\prime}(t)\right|\left|\int_{\mathbb{R}} z \partial_{c} \tilde{u} \psi_{A_{0}}\right|+K^{*} \varepsilon^{1 / 2} \int_{\mathbb{R}} z^{2}(t) e^{-\frac{1}{A_{0}}|y|} \\
& \leq \frac{\delta_{0}}{100} \int_{\mathbb{R}} z^{2}(t) e^{-\frac{1}{A_{0}}|y|}+K K^{*} A_{0} \varepsilon^{5 / 2} e^{-\varepsilon \gamma|\rho(t)|} .
\end{aligned}
$$

On the other hand, the terms (4.17) and (4.18) goes similarly to the terms $B_{1}$ and $B_{2}$ in Appendix B of [22. Indeed, we have

$$
\begin{aligned}
(4.17)+(4.18)= & -\int_{\mathbb{R}} \psi_{A_{0}}^{\prime}\left(3 z_{x}^{2}+c z^{2}-p Q_{c}^{p-1} z^{2}\right)-p \int_{\mathbb{R}}\left(Q_{c}^{p-1}\right)^{\prime} z^{2} \psi_{A_{0}} \\
& +\int_{\mathbb{R}} z^{2} \psi_{A_{0}}^{(3)}-2 \rho_{1}^{\prime}(t) \int_{\mathbb{R}} z \partial_{\rho} \tilde{u} \psi_{A_{0}} \\
& +2 p \int_{\mathbb{R}}\left(z \psi_{A_{0}}\right)_{x} z\left(R^{p-1}-Q_{c}^{p-1}\right)-\varepsilon f_{2} \int_{\mathbb{R}} z^{2} \psi_{A_{0}}^{\prime} .
\end{aligned}
$$

We finally get, taking $\varepsilon$ small, depending on $A_{0}$,

$$
\text { (4.17) }+ \text { (4.18) } \leq-\frac{\delta_{0}}{10} \int_{\mathbb{R}}\left(z_{x}^{2}+z^{2}\right)(t) e^{-\frac{1}{A_{0}}|y|} \text {. }
$$

Finally, the term (4.21) can be estimated as follows

$$
\begin{aligned}
|[4.21)| \lesssim & \left|\int_{\mathbb{R}} z\left(z \psi_{A_{0}}(y)\right)_{x}\left(\tilde{u}^{p-1}-R^{p-1}\right)\right|+\left|\int_{\mathbb{R}} z \psi_{A_{0}} \tilde{S}[\tilde{u}]\right| \\
\lesssim & \left|\int_{\mathbb{R}} z^{2} \psi_{A_{0}}^{\prime}(y)\left(\tilde{u}^{p-1}-R^{p-1}\right)\right| \\
& \quad+\left|\int_{\mathbb{R}} z z_{x} \psi_{A_{0}}(y)\left(\tilde{u}^{p-1}-R^{p-1}\right)\right|+A_{0}\left(K^{*}\right)^{2} \varepsilon^{5 / 2} e^{-\varepsilon \gamma|\rho(t)|} \\
\lesssim & A_{0} \varepsilon \int_{\mathbb{R}}\left(z^{2}(t)+z_{x}^{2}(t)\right) e^{-\frac{1}{A_{0}}|y|}+A_{0}\left(K^{*}\right)^{2} \varepsilon^{5 / 2} e^{-\varepsilon \gamma|\rho(t)|} .
\end{aligned}
$$

We have used that $\psi_{A_{0}}$ decreases exponentially as $y \rightarrow-\infty$, and (3.47). Collecting these estimates, we finally get (4.16). 
Corollary 4.5. One has, from 4.11) and 4.16),

$$
\int_{0}^{t}\left|c_{1}^{\prime}(s)\right| d s \lesssim K^{*} \varepsilon
$$

for all $t \in\left[0, T^{*}\right]$, by taking $A_{0}$ large enough, independent of $\varepsilon$ and $K^{*}$.

The main part of the proof is the introduction of the following Lyapunov functional $([23,28])$ : Let

$$
\mathcal{F}(t):=\frac{1}{2} \int_{\mathbb{R}}\left(z_{x}^{2}+c(t) z^{2}\right)-\frac{1}{p+1} \int_{\mathbb{R}}\left[(\tilde{u}+z)^{p+1}-\tilde{u}^{p+1}-(p+1) \tilde{u}^{p} z\right] .
$$

From [23] and the fact that $p<5$, there exists a constant, independent of $K^{*}$ and $\varepsilon$ such that for every $t \in\left[0, T^{*}\right]$

$$
\mathcal{F}(t) \gtrsim\|z(t)\|_{H^{1}(\mathbb{R})}^{2}
$$

The next step is to obtain independent estimates on $\mathcal{F}(t)$. We follow [29]. It is not difficult to check that

$$
\begin{aligned}
\mathcal{F}^{\prime}(t)= & -\int_{\mathbb{R}} z_{t}\left[z_{x x}-c z+(\tilde{u}+z)^{p}-\tilde{u}^{p}\right]+\frac{1}{2} c^{\prime}(t) \int_{\mathbb{R}} z^{2} \\
& -\int_{\mathbb{R}} \tilde{u}_{t}\left[(\tilde{u}+z)^{p}-\tilde{u}^{p}-p \tilde{u}^{p-1} z\right] .
\end{aligned}
$$

LEMma 4.6. There exists a constant $\gamma>0$ such that, for any $t \in\left[0, T^{*}\right]$,

$$
\begin{aligned}
\mathcal{F}(t)-\mathcal{F}(0) \lesssim & \left(K^{*}\right)^{4} \varepsilon^{2-\frac{1}{100}}+\left(K^{*}\right)^{3} \varepsilon^{\frac{3}{2}-\frac{1}{100}} \\
& +K^{*} \varepsilon+\int_{0}^{t} \varepsilon e^{-\varepsilon \gamma|\rho(s)|}\|z(s)\|_{H^{1}(\mathbb{R})}^{2} d s .
\end{aligned}
$$

Proof. Replacing (4.9) in 4.25) we get

$$
\begin{aligned}
& \mathcal{F}^{\prime}(t)= \\
& =\varepsilon \int_{\mathbb{R}} a_{0}^{\prime}(\varepsilon x) Q_{c_{0}} z\left[z_{x x}-c z+(\tilde{u}+z)^{p}-\tilde{u}^{p}\right]+c(t) \int_{\mathbb{R}}\left[(\tilde{u}+z)^{p}-\tilde{u}^{p}\right] z_{x} \\
& \quad-\rho_{1}^{\prime}(t) \int_{\mathbb{R}} \partial_{y} \tilde{u}\left[z_{x x}-c z+(\tilde{u}+z)^{p}-\tilde{u}^{p}\right] \\
& \quad+c_{1}^{\prime}(t) \int_{\mathbb{R}} \partial_{c} \tilde{u}\left[z_{x x}-c z+(\tilde{u}+z)^{p}-\tilde{u}^{p}\right] \\
& \quad+\int_{\mathbb{R}} \tilde{S}[\tilde{u}]\left[z_{x x}-c z+(\tilde{u}+z)^{p}-\tilde{u}^{p}\right]+\frac{1}{2} c_{1}^{\prime}(t) \int_{\mathbb{R}} z^{2}+\frac{1}{2} \varepsilon f_{1}(t) \int_{\mathbb{R}} z^{2} \\
& \quad-\int_{\mathbb{R}} \tilde{u}_{t}\left[(\tilde{u}+z)^{p}-\tilde{u}^{p}-p \tilde{u}^{p-1} z\right] .
\end{aligned}
$$

We consider the case $p=2$, the other cases being similar (see 28] for more details). First of all, note that

$$
\left|\varepsilon \int_{\mathbb{R}} a_{0}^{\prime}(\varepsilon x) Q_{c_{0}} z\left[z_{x x}-c z+(\tilde{u}+z)^{p}-\tilde{u}^{p}\right]\right| \lesssim \varepsilon e^{-\gamma \varepsilon\left|\rho_{0}(t)\right|}\|z(t)\|_{H^{1}(\mathbb{R})}^{2} .
$$


Next, after some simplifications, we get

$$
c(t) \int_{\mathbb{R}}\left[(\tilde{u}+z)^{p}-\tilde{u}^{p}\right] z_{x}=c(t) \int_{\mathbb{R}}\left[2 \tilde{u} z+z^{2}\right] z_{x}=-c(t) \int_{\mathbb{R}} \tilde{u}_{x} z^{2} .
$$

Now we estimate (4.28). Since $\partial_{y} \tilde{u}=Q_{c}^{\prime}+O\left(\eta_{\varepsilon} w_{y}\right)+O_{H^{1}(\mathbb{R})}\left(\varepsilon^{3 / 2} e^{-\varepsilon \gamma|\rho(t)|}\right)$ (cf. Proposition 3.77, one has

$$
\begin{aligned}
(14.28) & =-\rho_{1}^{\prime}(t) \int_{\mathbb{R}} \partial_{y} \tilde{u}\left[z_{x x}-c z+2 \tilde{u} z+z^{2}\right] \\
& =-\rho_{1}^{\prime}(t) \int_{\mathbb{R}} \tilde{u}_{x} z^{2}+O\left(\varepsilon e^{-\varepsilon \gamma|\rho(t)|}\|z(t)\|_{L^{2}(\mathbb{R})}^{2}\right) .
\end{aligned}
$$

Similarly, we have from (4.6)

$$
\begin{aligned}
(4.29)=c_{1}^{\prime}(t) & \int_{\mathbb{R}} \partial_{c} \tilde{u}\left[z_{x x}-c z+2 \tilde{u} z+z^{2}\right]=c_{1}^{\prime}(t) \int_{\mathbb{R}} \partial_{c} \tilde{u} z^{2} \\
& +O\left(\varepsilon e^{-\varepsilon \gamma|\rho(t)|}\|z(t)\|_{L^{2}(\mathbb{R})}^{2}+\varepsilon^{1 / 2}\left|c_{1}^{\prime}\right| e^{-\varepsilon \gamma|\rho(t)|}\|z(t)\|_{L^{2}(\mathbb{R})}\right) .
\end{aligned}
$$

On the one hand, we have from (3.47) and (4.8),

$$
\begin{aligned}
\mid \int_{\mathbb{R}} \tilde{S} & {[\tilde{u}]\left\{z_{x x}-c z+2 \tilde{u} z+z^{2}\right\} \mid \lesssim } \\
& \lesssim\left|\int_{\mathbb{R}} \partial_{x} \tilde{S}[\tilde{u}] z_{x}\right|+\left(1+K^{*} \varepsilon^{1 / 2}\right)\left|\int_{\mathbb{R}} \tilde{S}[\tilde{u}] z\right|+\left|\int_{\mathbb{R}} \tilde{S}[\tilde{u}] \tilde{u} z\right| \\
& \lesssim\left|\int_{\mathbb{R}} \partial_{x} \tilde{S}[\tilde{u}] z_{x}\right|+\left(1+K^{*} \varepsilon^{1 / 2}\right)\left|\int_{\mathbb{R}} \tilde{S}[\tilde{u}] z\right| \\
& \lesssim K^{*} \varepsilon^{2} e^{-\varepsilon \gamma|\rho(t)|}+\left(K^{*}\right)^{2} \varepsilon^{3 / 2} e^{-\varepsilon \gamma|\rho(t)|}\left[\int_{\mathbb{R}} e^{-\gamma_{0}|y|} z^{2}\right]^{1 / 2} .
\end{aligned}
$$

Concerning the second and third terms in (4.30),

$$
\frac{1}{2} c_{1}^{\prime}(t) \int_{\mathbb{R}} z^{2}+\frac{1}{2} \varepsilon f_{1}(t) \int_{\mathbb{R}} z^{2} \lesssim\left(\left|c_{1}^{\prime}(t)\right|+\varepsilon e^{-\varepsilon \gamma|\rho(t)|}\right)\|z(t)\|_{L^{2}(\mathbb{R})}^{2} .
$$

Finally,

$$
\begin{aligned}
\text { (4.31) }= & -\int_{\mathbb{R}}\left(\tilde{u}_{t}+\rho^{\prime} \tilde{u}_{x}-c^{\prime} \partial_{c} \tilde{u}\right) z^{2}+\rho^{\prime} \int_{\mathbb{R}} \tilde{u}_{x} z^{2} \\
& -c^{\prime} \int_{\mathbb{R}} \partial_{c} \tilde{u} z^{2}+O\left(\varepsilon e^{-\varepsilon \gamma|\rho(t)|}\|z(t)\|_{L^{2}(\mathbb{R})}^{2}\right) .
\end{aligned}
$$

We obtain

$$
\begin{gathered}
\mathcal{F}^{\prime}(t) \lesssim\left|c_{1}^{\prime}(t)\right|\|z(t)\|_{L^{2}(\mathbb{R})}^{2}+\varepsilon e^{-\gamma \varepsilon|\rho(t)|}\|z(t)\|_{L^{2}(\mathbb{R})}^{2}+\varepsilon\|z(t)\|_{H^{1}(\mathbb{R})}^{3}+K^{*} \varepsilon^{2} e^{-\varepsilon \gamma|\rho(t)|} \\
+\left(K^{*}\right)^{2} \varepsilon^{3 / 2} e^{-\varepsilon \gamma|\rho(t)|}\left[\int_{\mathbb{R}} e^{-\gamma 0|y|} z^{2}\right]^{1 / 2} .
\end{gathered}
$$

Using (4.22), we finally get after integration in time (here we use the condition $t \leq$ $\left.T^{*} \lesssim \varepsilon^{-1-2 \delta_{0}}\right)$

$$
\begin{aligned}
\mathcal{F}(t)-\mathcal{F}(0) \lesssim\left(K^{*}\right)^{3} \varepsilon^{\frac{3}{2}-\delta_{0}}+\left(K^{*} \varepsilon\right)^{2}+\int_{0}^{t} \varepsilon e^{-\gamma \varepsilon|\rho(s)|}\|z(s)\|_{H^{1}(\mathbb{R})}^{2} d s \\
+\left(K^{*}\right)^{2} \varepsilon^{3 / 2} \int_{0}^{t} e^{-\varepsilon \gamma|\rho(s)|}\left[\int_{\mathbb{R}} e^{-\gamma_{0}|y|} z^{2}\right]^{1 / 2} d s .
\end{aligned}
$$


Note that thanks to the Cauchy-Schwarz inequality and Lemma 4.4, we have

$$
\left(K^{*}\right)^{2} \varepsilon^{3 / 2} \int_{0}^{t} e^{-\varepsilon \gamma|\rho(s)|}\left[\int_{\mathbb{R}} e^{-\gamma_{0}|y|} z^{2}\right]^{1 / 2} d s \lesssim\left(K^{*}\right)^{2} \varepsilon^{3 / 2},
$$

for $\varepsilon$ small (depending on $K^{*}$ ). Indeed, we just need to justify that $\left|\int_{0}^{t} \varepsilon e^{-\gamma \varepsilon|\rho(s)|} d s\right| \lesssim$ 1 , independent of $\varepsilon$ and $K^{*}$. It is not difficult to see that the estimate above holds since $\rho^{\prime}(s) \geq \frac{9}{10} c(s) \geq \frac{8}{10} \min \left\{c_{f}, 1\right\}>0$. Therefore

$$
\mathcal{F}(t)-\mathcal{F}(0) \lesssim\left(K^{*}\right)^{3} \varepsilon^{\frac{3}{2}-\delta_{0}}+\left(K^{*} \varepsilon\right)^{2}+\int_{0}^{t} \varepsilon e^{-\gamma \varepsilon|\rho(s)|}\|z(s)\|_{H^{1}(\mathbb{R})}^{2} d s,
$$

as desired. The cases $p=3$ and 4 are similar. $\square$ We are finally in position to improve (4.4). Indeed, since from Lemma 4.3, $\mathcal{F}(0) \lesssim \varepsilon$, using (4.24) and Lemma (4.26) we get

$$
\begin{aligned}
\|z(t)\|_{L^{2}(\mathbb{R})}^{2} \lesssim & \varepsilon+\left(K^{*}\right)^{4} \varepsilon^{2-\delta_{0}}+\left(K^{*}\right)^{3} \varepsilon^{\frac{3}{2}-\delta_{0}}+\left(K^{*}\right)^{2} \varepsilon^{2} \\
& +\int_{0}^{t} \varepsilon e^{-\gamma \varepsilon|\rho(s)|}\|z(s)\|_{H^{1}(\mathbb{R})}^{2} d s .
\end{aligned}
$$

Now, by Gronwall's inequality (see e.g. [28] for a detailed proof),

$$
\|z(t)\|_{H^{1}(\mathbb{R})}^{2} \lesssim \varepsilon+\left(K^{*}\right)^{3} \varepsilon^{\frac{3}{2}-\delta_{0}} .
$$

with constant independent of $K^{*}$ and $\varepsilon$.

Let us come back to the main proof. From estimate (4.36), and taking $\varepsilon$ small, and $K^{*}$ large enough, we obtain that for all $t \in\left[0, T^{*}\right]$,

$$
\|z(t)\|_{H^{1}(\mathbb{R})}^{2} \leq \frac{1}{4}\left(K^{*}\right)^{2} \varepsilon .
$$

Therefore, we improve the estimate on $z(t)$ (4.8), and therefore (4.4)-(4.5) are also improved. The proof of Proposition 4.1 is complete. $\square$

5. Proof of the Main Theorems. We are now in position to give a direct proof of Theorem 1.2. The proof is very similar to the corresponding proof of Lemma 2.1. Indeed, we have (4.1) for all time $t \in[0, T]$; in particular, at $t=T$ one has

$$
\|u(T)-\tilde{u}(T, c(T), \rho(T))\|_{H^{1}(\mathbb{R})} \lesssim \sqrt{\varepsilon} .
$$

Note that, from (4.11) and (4.10), since $c(t)>c_{m}$ by (2.5),

$$
\begin{aligned}
c^{-\frac{1}{p-1}}(t) c^{\prime}(t)= & -\varepsilon \lambda_{p} a_{0}^{\prime}(\varepsilon \rho(t)) c(t)+c^{-\frac{1}{p-1}}(t) c_{1}^{\prime}(t) \\
= & -\varepsilon \lambda_{p} a_{0}^{\prime}(\varepsilon \rho(t)) \rho^{\prime}(t)+\varepsilon^{2} \lambda_{p} a_{0}^{\prime}(\varepsilon \rho(t)) f_{2}(t) \\
& +\varepsilon \lambda_{p} a_{0}^{\prime}(\varepsilon \rho(t)) \rho_{1}^{\prime}(t)+c^{-\frac{1}{p-1}}(t) c_{1}^{\prime}(t) .
\end{aligned}
$$

Hence, if $p=2$, and using (4.22),

$$
\log c(t)-\log c(0)=-\lambda_{2}\left[a_{0}(\varepsilon \rho(t))-a_{0}(\varepsilon \rho(0))\right]+O(\sqrt{\varepsilon}),
$$


from which we obtain

$$
c(t)=e^{-\lambda_{2} a_{0}(\varepsilon \rho(t))}(1+O(\sqrt{\varepsilon})), \quad p=2 .
$$

Similarly, if $p=3$ or 4 ,

$$
c(t)=\left[1-\lambda_{p} \frac{(p-2)}{p-1} a_{0}(\varepsilon \rho(t))\right]^{\frac{p-1}{p-2}}(1+O(\sqrt{\varepsilon})) .
$$

Now, we perform a detailed asymptotic analysis of $(c(t), \rho(t))$. First of all, note that from (5.2)-(5.3), $c(t)$ is strictly positive for all time. Indeed, the case $p=2$ is direct, and for $p=3$ or $p=4$, we have $c(t) \geq 1-K \sqrt{\varepsilon}$ in the case where $a_{\infty}$ is negative (or $c_{f}>1$, see (2.2) and (2.3)), and

$$
c(t) \geq\left[1-\lambda_{p} \frac{(p-2)}{p-1}\left\|a_{0}\right\|_{\infty}\right]^{\frac{p-1}{p-2}}-K \sqrt{\varepsilon}
$$

for the case $a_{\infty}>0\left(c_{f}<1\right)$. From (2.4) we have $\left\|a_{0}\right\|_{\infty}=a_{\infty}=\frac{(p-1)}{\lambda_{p}(p-2)}\left(1-c_{f}^{\frac{p-2}{p-1}}\right)$. Therefore,

$$
c(t) \geq c_{f}-K \sqrt{\varepsilon}>0 .
$$

We conclude that, for $\varepsilon>0$ small, $c(t) \geq \frac{99}{100} \min \left\{1, c_{f}\right\}>0$. Hence $\rho(t)$ is increasing and $\rho(t)-\rho(0) \geq \frac{99}{100} \min \left\{1, c_{f}\right\} t$, which implies that $\rho(T) \gtrsim T$. Moreover, from (2.3), $a(\varepsilon \rho(T))=a_{\infty}+O\left(\varepsilon^{10}\right)$. Taking $t=T$ in (5.2) and (5.3), we have

$$
c(T)=e^{-\lambda_{2} a_{\infty}}(1+O(\sqrt{\varepsilon}))=c_{f}(1+O(\sqrt{\varepsilon})), \quad p=2,
$$

and

$$
c(T)=\left[1-\lambda_{p} \frac{(p-2)}{p-1} a_{\infty}\right]^{\frac{p-1}{p-2}}(1+O(\sqrt{\varepsilon}))=c_{f}(1+O(\sqrt{\varepsilon})), \quad p=3,4,
$$

as desired. Finally, from (3.3), one has

$$
\left\|\tilde{u}(T, c(T), \rho(T))-Q_{c_{f}}(\cdot-\rho(T))\right\|_{H^{1}(\mathbb{R})} \lesssim \sqrt{\varepsilon} .
$$

Using (5.1) and the triangle inequality, the first estimate in (1.9) follows. Concerning the second one, it is a consequence of (4.10).

Finally, Corollary 1.3 is just a consequence of the behavior of $c(t)$ in (5.2), (5.3) and (2.3).

\section{REFERENCES}

[1] T. B. Benjamin, The stability of solitary waves, Proc. Roy. Soc. London A 328, (1972) 153-183.

[2] K. Beauchard and M. Mirrahimi, Practical stabilization of a quantum particle in a onedimensional infinite square potential well, SIAM J. Control Optim., 48 (2009), no. 2, p. $1179-1205$.

[3] K. Beauchard and J.M. Coron, Controllability of a quantum particle in a moving potential well, J. Funct. Anal. 232 (2006), pp. 328-389.

[4] J. L. Bona, P. Souganidis, and W. Strauss, Stability and instability of solitary waves of Korteweg-de Vries type, Proc. Roy. Soc. London 411 (1987), 395-412. 
[5] J. L. Bona, and B.Y. Zhang, The initial-value problem for the forced Korteweg-de Vries equation, Proc. Roy. Soc. Edinburgh Sect. A 126 (1996), no. 3, 571-598.

[6] J.-M. Coron and E. Crépeau, Exact boundary controllability of a nonlinear KdV equation with critical lengths, J. Eur. Math. Soc., 6 (2004), pp. 367-398.

[7] E. Cerpa, Exact controllability of a nonlinear Korteweg-de Vries equation on a critical spatial domain, SIAM J. Control Optim., 46 (2007), 877-899.

[8] E. Cerpa and E. Crépeau, Boundary controllability for the nonlinear Korteweg-de Vries equation on any critical domain, Ann. Inst. H. Poincaré Anal. Non Linéaire, 26 (2009), pp. 457-475.

[9] E. Crepeau, Exact controllability of the Korteweg-de Vries equation around a non-trivial stationary solution, Internat. J. Control, 74 (2001), pp. 1096-1106.

[10] R. Grimshaw, Slowly varying solitary waves. I. Korteweg-de Vries equation, Proc. Roy. Soc. London Ser. A 368 (1979), 359-376.

[11] J. Holmer, Dynamics of KdV solitons in the presence of a slowly varying potential, Int. Math. Res. Not. IMRN 2011, no. 23, 5367-5397.

[12] J. Holmer, G. Perelman, and M. Zworski, Effective dynamics of double solitons for perturbed $m K d V$, Comm. Math. Phys. 305 (2011), no. 2, 363-425.

[13] D. J. Kaup, and A. C. Newell, Solitons as particles, oscillators, and slowly changing media: a singular perturbation theory, Proc. Roy. Soc. London Ser. A 361 (1978), 413-446.

[14] C. E. Kenig, G. Ponce, and L. Vega, Well-posedness and scattering results for the generalized Korteweg-de Vries equation via the contraction principle, Comm. Pure Appl. Math. 46, (1993) 527-620.

[15] K. Ko and H. H. Kuehl, Korteweg-de Vries soliton in a slowly varying medium, Phys. Rev. Lett. 40 (1978), no. 4, 233-236.

[16] H. Lange, and H. Teismann, Controllability of the nonlinear Schrödinger equation in the vicinity of the ground state, Math. Meth. Appl. Sci. 30 (2007) 1483-1505.

[17] C. Laurent, L. Rosier, and B.Y. Zhang, Control and stabilization of the Korteweg-de Vries equation on a periodic domain, Comm. Partial Differential Equations 35 (2010), no. 4, $707-744$.

[18] P. Lochak, On the adiabatic stability of solitons and the matching of conservation laws, J. Math. Phys. 25 (1984), no. 8, 2472-2476.

[19] F. Linares, and A. Pazoto, On the exponential decay of the critical generalized Korteweg-de Vries with localized damping, Proc. Amer. Math. Soc., 135 (2007), pp. 1515-1522.

[20] F. Linares, and A. Pazoto, Asymptotic behavior of the Korteweg-de Vries equation posed in a quarter plane, J. Differential Equations 246 (2009), no. 4, 1342-1353.

[21] Y. Martel, and F. Merle, Blow up in finite time and dynamics of blow up solutions for the $L^{2}$-critical generalized KdV equation, J. Amer. Math. Soc. 15 (2002), no. 3, 617-664.

[22] Y. Martel and F. Merle, Asymptotic stability of solitons of the subcritical gKdV equations revisited, Nonlinearity 18 (2005) 55-80.

[23] Y. Martel and F. Merle, Description of two soliton collision for the quartic gKdV equations, Ann. of Math. (2) 174 (2011), no. 2, 757-857.

[24] Y. Martel and F. Merle, Inelastic interaction of nearly equal solitons for the quartic gKdV equation, Invent. Math. 183 (2011), no. 3, 563-648.

[25] C. P. Massarolo, G. P. Menzala, and A. Pazoto, On the uniform decay for the Korteweg-de Vries equation with weak damping, Math. Methods Appl. Sci. 30 (2007), no. 12, 1419-1435.

[26] F. Merle, and L. Vega, $L^{2}$ stability of solitons for the KdV equation, Int. Math. Res. Notices 2003, no. 13, 735-753.

[27] M. Mirrahimi, Lyapunov control of a quantum particle in a decaying potential, Ann. I.H.P. (c) Nonlinear Analysis, 26 (2009), pp. 1743-1765.

[28] C. Muñoz, On the soliton dynamics under slowly varying medium for generalized Kortewegde Vries equations, Anal. PDE 4 (2011), no. 4, 573-638.

[29] C. Muñoz, Dynamics of soliton-like solutions for slowly varying, generalized gKdV equations: refraction vs. reflection, SIAM J. Math. Anal. 44 (2012), no. 1, 1-60.

[30] A. Pazoto, L. Rosier, Uniform stabilization in weighted Sobolev spaces for the KdV equation posed on the half-line, Discrete Contin. Dyn. Syst. Ser. B 14 (2010), no. 4, 1511-1535.

[31] A. Pazoto, M. Sepúlveda, and O. Vera Villagrán, Uniform stabilization of numerical schemes for the critical generalized Korteweg-de Vries equation with damping, Numer. Math. 116 (2010), no. 2, 317-356.

[32] Q. Lin, Soliton dynamics for a non-hamiltonian perturbation of $m K d V$, Differential Integral Equations 26 (2013), no. 1-2, 81-104.

[33] L. Rosier, Exact boundary controllability for the Korteweg-de Vries equation on a bounded domain, ESAIM Control Optim. Calc. Var., 2 (1997), pp. 33-55. 
[34] L. Rosier, Exact boundary controllability for the linear Korteweg-de Vries equation on the half-line, SIAM J. Control Optim. 39 (2000), no. 2, 331-351.

[35] L. Rosier, and B.Y. Zhang, Global stabilization of the generalized Korteweg-de Vries equation posed on a finite domain, SIAM J. Control Optim. 45 (2006), no. 3, 927-956.

[36] D. L. Russell and B.Y. Zhang, Controllability and stabilizability of the third-order linear dispersion equation on a periodic domain, SIAM J. Control Optim., 31 (1993), pp. 659-676.

[37] D. L. Russell, and B.Y. Zhang, Exact controllability and stabilizability of the Korteweg-de Vries equation, Trans. Amer. Math. Soc. 348 (1996), no. 9, 3643-3672.

[38] D. L. Russell and B.Y. Zhang, Smoothing and decay properties of the Korteweg-de Vries equation on a periodic domain with point dissipation, J. Math. Anal. Appl., 190 (1995), pp. $449-488$.

[39] S. M. Sun, The Korteweg-de Vries equation on a periodic domain with singular-point dissipation, SIAM J. Control Optim., 34 (1996), pp. 892-912.

[40] M.I. Weinstein, Modulational stability of ground states of nonlinear Schrödinger equations, SIAM J. Math. Anal. 16 (1985), no. 3, 472-491.

[41] B. Y. Zhang, Exact boundary controllability of the Korteweg-de Vries equation, SIAM J. Control Optim. 37 (1999), no. 2, 543-565. 